Federal Reserve Bank of Minneapolis

Research Department Staff Report 245

March 1998

\title{
A Gain From Trade: More Research, Less Obstruction*
}

\author{
Thomas J. Holmes \\ University of Minnesota \\ James A. Schmitz, Jr. \\ Federal Reserve Bank of Minneapolis
}

\begin{abstract}
There is an old wisdom that reductions in tariffs force changes on producers that lead to costless, or nearly so, increases in productivity. We construct a technology-ladder model that captures this wisdom. As in other technology-ladder models, time spent in research helps propel an industry up a technology-ladder. In contrast to the literature, we include another activity that plays a role in determining an industry's position on the technology-ladder: attempts to obstruct the research program of rivals (through regulations, for example). In this world, reductions in tariffs between countries lead producers to spend more time in research and less in obstruction of rivals.

${ }^{*}$ The views expressed herein are those of the authors and not necessarily those of the Federal Reserve Bank of Minneapolis or the Federal Reserve System.
\end{abstract}




\section{Introduction}

There is an old wisdom, aptly expressed by Balassa (1967), that "intensified competition [resulting from tariff reductions] may have beneficial effects on the national economies of the trading countries through 'forced' improvements in manufacturing efficiency." He goes on to argue that "gains of this sort [include] ...[the] 'challenge' or 'salutary jolt' that would bring about a change in the outlook of business and provide an inducement for a transition from the 'traditional ways of doing things'..."(Balassa 1967, pp. 105-6). Implicit in this wisdom is that the changes that are forced on producers are costless, or nearly so, to implement. ${ }^{1}$

Industry case studies lend some support to this view. For example, the view that openness to trade spurs the productivity of domestic producers is a theme that emerges from the cross-country productivity studies conducted by McKinsey and Company (Manufacturing Productivity 1993). (See Baily and Gersbach (1995) for a discussion of the McKinsey study.) In summarizing the automobile case study, the report argues that

Direct competition from leading edge auto producers in Japan has caused the exposed U.S. auto industry to respond in kind. The German auto industry, largely sheltered from Japanese competition, has not-productivity in the German industry is significantly lower than that in both Japan and the U.S. (Manufacturing Productivity 1993, Executive Summary, p. 3). ${ }^{2}$

But while this view that reduced trade protection can jolt domestic producers is widespread and case studies lend some support to it, the phenomenon "has no place in traditional

\footnotetext{
${ }^{1} \mathrm{~A}$ similar view also has a long history in the industrial organization literature. In that literature, the wisdom concerns the effects of increased competition on the productivity of a monopolist. See, for example, Leibenstein (1966).

${ }^{2}$ As is well known, the United States industry improved its productivity by adopting new production methods developed in Japan. The Europeans were slower to adopt the methods.
} 
theory" (Balassa 1967, p. 105). It had no place in traditional theory in 1967, nor does it 30 years later. The purpose of this paper is to offer a concrete channel through which lower tariffs can jolt producers to change their behavior and costlessly increase productivity and to develop this channel in a formal model.

The model we construct below is a technology-ladder model (as in Grossman and Helpman (1991)). As in other technology-ladder models, time spent in research helps propel an industry up a technology-ladder. In contrast to the literature, we include another activity that plays a role in determining an industry's position on the technology-ladder: attempts to obstruct a rival's research program. This activity is widespread. The activity includes attempts to install regulations that prohibit the use of new technology discovered by rivals. ${ }^{3}$

Our results can be summarized as follows. In the model, lower tariffs around the world change the returns associated with the two activities, research and obstruction. As our title suggests, lower tariffs tilt the returns from obstruction to research, leading entrepreneurs/firms to shift their effort from obstructing the research progress of rivals to their own research programs. This change in behavior leads to a costless increase in productivity throughout the world.

Let us briefly explain the model. Consider first a country that is in isolation (say, it has infinite tariffs). There are two goods: a service good, produced under constant returns

\footnotetext{
${ }^{3}$ Let us give a few concrete examples of the activity. In the telecommunications industry, many recent technological breakthroughs have threatened existing technologies. The users of existing technologies have often successfully put restrictions on these new technologies. When cable TV emerged, network TV succeeded in pushing for restrictions on cable operators (for example, that they carry certain channels). As direct TV emerges, cable TV has pushed for restrictions on these broadcasters. Another example is Mars's recent successful lobbying campaign that halted the sale of a new candy by Nestlé in the United States. (See the article "Bittersweet" in The New Republic, October 27, 1997.) These two episodes are examples of what industrial organization economists have coined "regulatory capture." Our guess is that much of the activity labeled regulatory capture is directed at this activity of limiting the technology of rivals (either inside or outside the industry).
} 
by competitive producers, and a manufactured good, produced by two entrepreneurs/firms. The manufacturing technology can be improved. In particular, there is a technology-ladder in manufacturing, with each rung on the ladder an improvement over the previous rung. The two entrepreneurs vie for technology leadership. Both entrepreneurs have a fixed unit time endowment that they devote to ascending to and maintaining technology leadership.

The entrepreneur who has the best technology at a given date we call the leader, and he or she earns a monopoly return as long as his or her technology is best. The entrepreneur who is behind at a given date is called the follower. The follower spends his or her entire time endowment pursuing research in attempting to discover the next stage technology. The leader divides his or her time endowment between two activities. The leader, too, can do research to introduce an improvement, but recognizing that his or her research may fail, the leader can also expend effort to obstruct the progress of the follower in the event that the follower's research succeeds.

The leader, in choosing how to divide time between research and obstruction, compares two values: the value to being the leader at the next rung on the ladder and the value to being the leader at the current ladder rung. The return to time spent in research depends on the value to being the leader at the next rung (since research moves the entrepreneur up the ladder), while the return to time spent in obstruction depends on the value to being the leader at the current rung (since the return to obstruction comes only from protecting the current monopoly return). The greater is the ratio of the value to being the leader at the next rung to that of the value to being the leader at the current rung, the greater is the amount of time spent in research (and the less is the time spent in obstruction).

Next, consider a world that is composed of a large number of countries that are identical 
to the single country above. Assume that households in a country have preferences over service goods, the local manufactured good, and foreign manufactured goods. The leader in a country earns profits by selling at home and abroad. Now, in choosing how to divide time between research and obstruction of the follower's progress, the leader again compares the value to being the leader at the next rung on the ladder with the value to being the leader at the current ladder rung. We show that, under some reasonable conditions described shortly, that the lower are tariffs, the greater is the value to being the leader at the next rung on the ladder relative to the value to being the leader at the current ladder rung. Hence, with lower tariffs, the leader in each country spends more time in research and less in obstruction of the follower. Productivity costlessly increases. Productivity increases for two reasons: there is more research in each country and the breakthroughs of followers are invalidated less frequently.

Let us now briefly explain conditions under which this key profit ratio, that is, the ratio of the value to being the leader at the next rung to that of the value to being the leader at the current rung, increases as tariffs are lowered. In the model, when a leader successfully makes a step up the ladder, the leader follows with a price cut. The greater is the elasticity of demand for the entrepreneur's product in the local and foreign markets, the greater is the increase in profit that follows this price cut (that is, the greater will be this key ratio). Here, let us simply explain conditions under which the lower is the tariff, the greater is the elasticity of demand for the local manufactured good. Suppose that the tariff is initially extremely large so that, approximately, households purchase only the local manufactured good and services. Then as the leader cuts price, households make substitutions from services to the local manufactured good. The elasticity of demand for the local manufactured good 
depends on the elasticity of substitution between the local manufactured good and services (and equals $\eta$ in the model). Next, suppose tariffs are low. Now when a leader makes a step up the ladder and cuts price, households make substitutions from services and foreign manufactured goods to the local manufactured good. If the elasticity of substitution between the local and foreign manufactured goods (which equals $\sigma$ in the model) is greater than the elasticity of substitution between the local manufactured good and services (that is, $\sigma>\eta$, which we assume), then demand for the local manufactured good is more elastic with lower tariffs. Hence, making a step up the ladder, and cutting price, leads to a larger increase in profits when tariffs are lower.

Regarding related literature, most discussion of this old wisdom has been informal, like that in Balassa above. However, Olson (1982) discusses in a rigorous way (though without a model) how competition between states in a federal system like the United States, where tariffs are zero, can limit the power and influence of special interest groups. In Holmes and Schmitz (1995), we show that reductions in tariffs can lead a special interest group to drop its resistance to a new technology. In other words, the group drops its obstruction activities. This earlier paper shares much in spirit with this current paper. However, the earlier paper does not model research effort and the evolution of new technology. In this regard, the current paper is a big improvement on the earlier one. It also is of interest to briefly compare our results with those in the industrial organization literature examining market structure and research. It will be best to discuss those papers after we develop our model.

The rest of the paper proceeds as follows. The model is described in the next section. Our definition of equilibrium is given in Section 3. Section 4 presents a characterization of equilibrium. Section 5 presents the main proposition that reductions in tariffs lead to 
increases in research. In the last section, we return to discuss some of the reasons for the assumptions we make about the model in Section 2. We also discuss some related literature in this closing section.

\section{Model}

Time is discrete; $t \in\{0,1,2, \ldots\}$. Countries (or locations) are indexed by $i$, where $i \in[0,1]$. At each location, there is a unit measure of households and two entrepreneurs indexed by $e \in\{1,2\}$. Households live a single period (being replaced by another unrelated household at the end of a period). Entrepreneurs live forever. Households and entrepreneurs are endowed with a unit of time each period. Both groups are immobile. At each location, there are two sectors: a manufacturing sector and a non-manufacturing sector. Let us, for simplicity, call the sector the service sector. We now turn to describe preferences and technologies.

Preferences. Households at location $j$ have a utility function

$$
u(x, y)=\left[(1-\alpha) x^{\frac{\eta-1}{\eta}}+\alpha y^{\frac{\eta-1}{\eta}}\right]^{\frac{\eta}{\eta-1}}
$$

where $x$ is units of a composite manufacturing good, $y$ is units of the service good, and $\eta$ is the elasticity of substitution between $x$ and $y$. The manufacturing composite is given by

$$
x=\left[(1-\lambda) x_{C}^{\frac{\sigma-1}{\sigma}}+\lambda x_{D}^{\frac{\sigma-1}{\sigma}}\right]^{\frac{\sigma}{\sigma-1}}
$$

where $x_{C}$ is a composite (" $C$ ") of foreign manufacturing goods and $x_{D}$ is the domestic (" $D$ ") manufacturing good. The composite $x_{C}$ satisfies

$$
x_{C}=\left[\int_{i \neq j} x(i)^{\frac{\sigma-1}{\sigma}} d i\right]^{\frac{\sigma}{\sigma-1}}
$$

where, again, $j$ denotes the location of the household, $\lambda \in(0,1)$, and $\sigma$ is the elasticity of substitution between $x_{C}$ and $x_{D}$ and between any two foreign manufactured goods. 
Regarding preference parameters, we assume that

$$
\sigma>1 \text { and } \sigma>\eta
$$

The first assumption, $\sigma>1$, implies that the domestic and foreign manufactured goods (say, cars) are reasonably good substitutes for each other. The second assumption, $\sigma>\eta$, means the domestic and foreign manufactured goods are better substitutes for each other than are the domestic manufactured good and the service good. These assumptions seem reasonable.

To keep the analysis as simple as possible, we assume that entrepreneur period-utility depends only on the service good and is denoted $u(y)=y$. Over the infinite-horizon, entrepreneurs have preferences given by $E\left(\sum_{t=0}^{\infty} \beta^{t} y_{t}\right)$.

Production Technologies. Household time is used to produce $x$ and $y$. The service good is produced by service firms that employ a constant returns to scale technology, with one unit of household time producing one unit of the service good. This technology is fixed through time.

Entrepreneurs produce the manufactured good with household time. The manufacturing technology differs through time. In any period, the technology is at one of three levels, or ladder rungs, indexed by $a \in\{0,1,2\} .{ }^{4}$ If the technology is at $a=0$, then the industry is in an immature phase. Both entrepreneurs produce with the technology

$$
x=n
$$

where $n$ is units of household time. If the technology is at a ladder rung $a>0$, then one of the entrepreneurs, whom we call the leader, produces with the technology associated with $a$,

\footnotetext{
${ }^{4}$ Formally, the technology is at a ladder rung. However, we will often use the terminology that the manufacturing industry, or simply industry, is at a ladder rung.
} 
namely,

$$
x=\gamma^{a} n
$$

while the other entrepreneur, whom we call the follower, produces with the technology a level below, namely,

$$
x=\gamma^{a-1} n
$$

where $\gamma>1$ and $a \in\{1,2\}$. We now turn to describe how the industry moves up the ladder and how the leader/follower status is determined.

Technology for Moving up the Ladder. As in other technology-ladder models, time spent in research, which we denote by $r$, helps propel an industry up the ladder. But in contrast to the literature, this paper includes another activity that plays a role in determining an industry's position on the ladder. Again, this activity is the attempt to obstruct a rival's research program. We denote the time spent in obstruction by $s$. We now give the details of how an industry's ladder position is determined.

Let us begin by assuming that at some date $t$, the industry is at ladder rung $a=1$. One of the entrepreneurs is the leader, the other the follower. Next period there are four possible industry positions: today's leader at $a=1$ is the leader at $a=2$, today's follower at $a=1$ is the leader at $a=2$, today's leader at $a=1$ remains the leader at $a=1$, and the industry returns to an immature phase, that is, $a=0$. The probability of each of these positions is as follows.

The probability that, entering next period, the industry returns to an immature phase is given by

$$
q_{B}=\delta
$$


where the subscript " $B$ " is for bottom of the ladder. ${ }^{5}$

Today's leader is the leader at $a=2$ tomorrow if he or she discovers $a=2$ and if the industry does not return to an immature phase. The probability that the leader discovers $a=2$ is given by $f(r)$, where $f(r)$ satisfies $f(0)=0, f(1)<1, f^{\prime}>0, f^{\prime \prime}<0$, and $\lim _{r \rightarrow 0} f^{\prime}(r)=\infty$. The probability that today's leader is the leader at $a=2$ tomorrow equals the probability that the leader is successful multiplied by the probability that the industry does not return to an immature phase, or

$$
q_{L}(r)=(1-\delta) f(r)
$$

where the subscript " $L$ " is for leader advances.

Today's follower spends his or her entire time endowment in research. Hence, $f(1)$ is the probability that the follower discovers $a=2$. The follower becomes the leader at $a=2$ if the leader's research fails, the follower's research succeeds, and the obstruction efforts of the leader fail. The leader attempts to obstruct the progress of the follower so that in the event the leader's research fails, the leader can maintain his or her current leadership. The probability that the leader obstructs the follower's progress is given by $g(s)$, where $s$ is time devoted to obstruction. (We assume that the properties listed above for the function $f(r)$ are also true for the function $g(s)$.) Note that it must be that $r+s=1$, since the leader has only a unit of time each period. Hence, the probability that the follower advances to leadership is

$$
q_{F}(r, s)=(1-\delta) f(1)[1-f(r)][1-g(s)]
$$

\footnotetext{
${ }^{5}$ An interpretation of this assumption is that are many potential manufactured goods that can be produced at a location. Each of these goods enters the utility function in the same manner. At a particular date, households desire to consume one of these goods. Entrepreneurs develop new methods of producing this good. Periodically there is a shock to preferences so that households now desire to consume some other good from this set. Hence, the industry must start from scratch in developing new methods for producing this good.
} 
where the subscript " $F$ " is for follower advances.

The probability that the leader maintains his or her position is given by

$$
q_{M}(r, s)=1-q_{L}(r)-q_{F}(r, s)-q_{B}
$$

where the subscript " $M$ " means that the status quo is maintained.

Next, suppose that at some date $t$, the industry is at ladder rung $a=2$. One of the entrepreneurs is the leader, the other the follower. The leader remains the leader at $a=2$ until the industry enters another immature phase (and therefore falls to the bottom of the ladder). The probability of entering an immature phase from $a=2$ is denoted $\delta$ (the same probability as entering the phase from $a=1$ ).

Finally, suppose that at date $t$, the industry is in an immature phase, that is, $a=0$. Both entrepreneurs spend their unit time endowment in research. An entrepreneur becomes the leader at $a=1$ tomorrow if his or her research is successful and the other's is not or if both are successful and he or she wins a coin toss. The probability of becoming the leader at $a=1$ tomorrow is

$$
q_{1}=(1-\delta)\left(f(1)(1-f(1))+\frac{1}{2} f(1)^{2}\right)
$$

where the subscript " 1 " refers to leadership at $a=1$. Note that $q_{1}$ depends only on model parameters.

Technology for Moving Goods. Service goods can be costlessly moved between countries. There is a tariff on manufactured goods produced at one location and sold at another. Tariff revenues are not returned to households or entrepreneurs. We describe these tariffs shortly. 


\section{Definition of Equilibrium}

In this section, we define a multi-country equilibrium for this environment. Let household time be the numeraire good. Households sell their time endowment to entrepreneurs and service firms. The wage $w$ paid in both sectors equals one. Households spend their unit income in a period to purchase services and manufactured goods (from domestic and foreign manufacturers). Entrepreneurs sell their product to households at home and abroad. They purchase services using current period profits.

Consider the state of the economy. Let $\mu_{a}$ be the fraction of countries at rung $a$ and $\mu=\left(\mu_{0}, \mu_{1}, \mu_{2}\right)$ the vector of rung proportions. The state of the economy is $\mu$. For an entrepreneur, the state is given by $(a, z, \mu)$, where $a$ is the ladder rung position in the country, $z$ is a variable indicating the entrepreneur's follower/leader status $(z \in\{F, L\}$ with " $F$ " denoting the follower, " $L$ " the leader), and $\mu$ is the aggregate state. Below, we focus on equilibria where entrepreneur and household choices do not vary over time and where those choices depend on the country only through the current position of the manufacturing technology on the technology ladder. In these equilibria, the manufacturing technology in a country moves up and down the ladder over time. However, at the world level, the distribution of countries over ladder rung positions is constant.

There are two parts to the definition of multi-country equilibrium given below. The first part defines what we mean by a research/obstruction equilibrium. The second part defines what we mean by a product-market equilibrium. We go over these definitions before presenting the definition of multi-country equilibrium.

\section{Part 1. Research/Obstruction Equilibrium}

In the equilibria we study, the profits (measured in numeraire) earned by entrepreneurs 
at a given ladder rung do not vary over time. The profits earned at $a=0$ (that is, during an immature phase of the industry) equal zero. The profits earned by a follower at $a=1$ and $a=2$ are both zero. We let $\pi_{1}$ and $\pi_{2}$ denote the profit earned by a leader at the respective ladder rungs. In the equilibria we study, these profits satisfy

$$
\pi_{1} \geq 0 \text { and } \pi_{2} \geq \pi_{1}
$$

Given a pair $\left(\pi_{1}, \pi_{2}\right)$ satisfying (11), we can define an infinite-horizon research/ obstruction game between the two entrepreneurs in a given country (or industry) starting at, say, $a=0$ at date $t=0$. The objective of each entrepreneur is to maximize the expected sum of discounted profits over the infinite-horizon. The choices of entrepreneur $e$ are simply what research level $r_{e t}$ to choose if $e$ is the leader at $a=1$ at date $t$ (where obstruction activities are given by $\left.s_{e t}=1-r_{e t}\right)$.

We focus on symmetric, Markov-perfect equilibria of this game. In order to define such equilibria, let $r$ denote the research choice of an entrepreneur who finds himself or herself the leader at $a=1$. Let $v_{a, z}$ be the expected sum of discounted profits of an entrepreneur who has status $z$ at rung $a$. When the industry is in an immature phase, there is no leader or follower, so both entrepreneurs have the same expected sum of discounted profits, which we denote $v_{0}$. Let $V=\left(v_{0}, v_{1, F}, v_{1, L}, v_{2, F}, v_{2, L}\right)$ denote the vector of values. We then have the following definition.

Definition 1. The pair $(r, V)$ is a symmetric, Markov-perfect equilibrium of the research/obstruction game if

$$
\begin{aligned}
& \text { - } r=\arg \max _{r \in[0,1]}\left[q_{L}(r) v_{2, L}+q_{F}(r, 1-r) v_{2, F}+q_{M}(r, 1-r) v_{1, L}+q_{B} v_{0}\right] \\
& \text { - } v_{1, L}=\pi_{1}+\beta\left[q_{L}(r) v_{2, L}+q_{F}(r) v_{2, F}+q_{M}(r) v_{1, L}+q_{B} v_{0}\right]
\end{aligned}
$$


- $v_{1, F}=0+\beta\left[q_{L}(r) v_{2, F}+q_{F}(r) v_{2, L}+q_{M}(r) v_{1, F}+q_{B} v_{0}\right]$

- $v_{0}=0+\beta\left[q_{1} v_{1, L}+q_{1} v_{1, F}+\left(1-2 q_{1}\right) v_{0}\right]$

- $v_{2, L}=\pi_{2}+\beta\left[(1-\delta) v_{2, L}+\delta v_{0}\right]$

- $v_{2, F}=0+\beta\left[(1-\delta) v_{2, F}+\delta v_{0}\right]$

where $q_{B}=\delta$ and where $q_{L}(r), q_{F}(r, s), q_{M}(r, s)$, and $q_{1}$ are given in (7), (8), (9), and (10), respectively.

For brevity's sake, we refer to this symmetric, Markov-perfect equilibrium as the research/obstruction equilibrium. We analyze this equilibrium in the next section.

\section{Part 2. Product-Market Equilibrium}

Given a research choice $r$ by the leader at $a=1$, there is an implied stationary vector of rung proportions $\mu$. Then, given the implied $\mu$, we can think of defining a currentperiod product market where entrepreneurs set prices to maximize current-period profits and households maximize utility by making choices given prices. A product-market equilibrium is a list of choices and prices such that household choices are utility maximizing, entrepreneur prices are profit maximizing, and today's markets clear. Given a product-market equilibrium, we can calculate the profits earned by a leader at each ladder rung.

In the equilibria we explore, leaders in an $a$-country set prices $p_{D, a}$ and $p_{E, a}$ (in units of numeraire) for their good in the domestic and foreign markets, respectively. ${ }^{6}$ If a good is sold to a household in a foreign market, then that household must pay $p_{E, a}(1+\tau)$ per unit for the good, where $\tau$ is the tariff on the good. Households form their demands given these

\footnotetext{
${ }^{6}$ If the technology is at ladder rung $a$ in some country, we will call that country an $a$-country.
} 
prices, $p_{D, a}$ and $p_{E, a}$, the service good price $p_{y}=1$, their unit income, and tariffs $\tau$. The leader and follower in a given country set prices by competing in a Bertrand fashion (with the leader having, of course, a lower marginal cost than the follower).

Given these preliminaries, we can give the following definition.

Definition 2. A list $\left(\left(\pi_{1}^{e}, \pi_{2}^{e}\right),\left(r^{e}, V^{e}\right), \mu^{e}\right)$ is a stationary, symmetric, multi-country equilibrium if (i) given $\left(\pi_{1}^{e}, \pi_{2}^{e}\right)$, the vector $\left(r^{e}, V^{e}\right)$ constitutes a research/obstruction equilibrium; and (ii) given $r^{e}$, the vector $\mu^{e}$ is the implied stationary distribution of rung proportions, and given $\mu^{e},\left(\pi_{1}^{e}, \pi_{2}^{e}\right)$ is the current period profit vector emerging from the product-market equilibrium.

For brevity's sake, we refer to this stationary, symmetric, multi-country equilibrium as the multi-country equilibrium. We show that the multi-country equilibrium exists, and we characterize it. We then compare two multi-country equilibria associated with two different tariff levels. We show that the level of research is higher (and, hence, the level of obstruction lower) in the equilibria associated with lower tariffs. Hence, the distribution of $\mu$ is greater.

\section{Characterization of Equilibrium}

In this section, we show that a multi-country equilibrium exists, and we characterize properties of the equilibrium. We do this in three steps (corresponding to the three subsections below). In the first subsection, we study the research/obstruction equilibrium. We show that the research choice $r$ in the research/obstruction equilibrium depends on profits only through the profit ratio $\pi=\frac{\pi_{2}}{\pi_{1}}$. We label this research level $r^{*}(\pi)$. In the second subsection, we study the product-market equilibrium. Given a research level $r$, we calculate the implied stationary distribution of $\mu$. Given this $\mu$, we calculate the level of profits, as well as the profit 
ratio, that emerges in the product-market equilibrium. We label this profit ratio $\pi^{* *}(r)$. In the third subsection, we show that a multi-country equilibrium corresponds to a pair $\left(\pi^{e}, r^{e}\right)$ such that $r^{e}=r^{*}\left(\pi^{e}\right)$ and $\pi^{e}=\pi^{* *}\left(r^{e}\right)$, and we show that such a pair always exists.

\section{A. The Research/Obstruction Equilibrium}

Recall that given a vector $\left(\pi_{1}, \pi_{2}\right)$ satisfying (11), we can define a research/obstruction game between the two entrepreneurs in a given country. This subsection shows that given such a pair $\left(\pi_{1}, \pi_{2}\right)$, there is a unique research/obstruction equilibrium. It also characterizes properties of the unique research choice.

In order to guarantee uniqueness of the research/obstruction equilibrium, we impose the following condition on the functions $f(\cdot)$ and $g(\cdot)$. For all $r \in(0,1)$,

$$
f^{\prime \prime}(r)[1-g(1-r)]+g^{\prime \prime}(1-r)[1-f(r)]+2 f^{\prime}(r) g^{\prime}(1-r) \leq 0 .
$$

This condition guarantees concavity of the leader's objective function in the choice $r$ (that is, the objective function given in the definition of research/obstruction equilibrium) and hence uniqueness of the research choice. ${ }^{7}$

The main result of this section is given in the following lemma.

Lemma. Assume that (12) holds. For a given $\left(\pi_{1}, \pi_{2}\right)$ satisfying (11), there exists a unique research/obstruction equilibrium. The research choice in the unique equilibrium, call

\footnotetext{
${ }^{7}$ An example of functions that satisfy this condition is as follows. Suppose that the research and obstruction functions are power functions and have the same form, namely, $f(r)=\theta r^{\zeta}$ and $g(s)=\theta s^{\zeta}$. The parameters $\theta$ and $\zeta$ must lie between zero and one. For these functions, condition (12) is satisfied if

$$
\theta \leq(1-\zeta) 2^{\zeta} .
$$

So, for example, if $\zeta=\frac{1}{2}$, then $\theta \leq 0.71$ is required to satisfy the concavity condition.
} 
it $r^{*}$, depends on the parameters $\pi_{1}$ and $\pi_{2}$ only through the ratio $\pi=\frac{\pi_{2}}{\pi_{1}}$. Let $r^{*}(\pi)$ denote this unique research level. Research $r^{*}(\pi)$ is strictly increasing in the ratio $\pi$.

The intuition for this result is as follows. That $r^{*}$ depends only on the ratio of leader profits is an artifact of the restriction that time must be divided between the two activities. In trying to see why research is increasing in the ratio, keep $\pi_{2}$ fixed and decrease $\pi_{1}$. As $\pi_{1}$ gets smaller, the status quo is worth less. Hence, $r$ increases.

Here is a sketch of the proof of the preceding lemma. Consider the following problem: call it problem $\mathcal{P}$. Suppose that the industry is at $a=1$. Suppose that the leader chooses $r$ today to maximize the expected sum of discounted profits, given $\left(\pi_{1}, \pi_{2}\right)$, and subject to the constraint that any future leader at $a=1$ is constrained to choose $r=\tilde{r}$. In order to formally state this problem, let $\tilde{V}=\left(\tilde{v}_{0}, \tilde{v}_{1, F}, \tilde{v}_{1, L}, \tilde{v}_{2, F}, \tilde{v}_{2, L}\right)$ denote the vector of values for being at ladder rung $a$ with status $z$ if $r=\tilde{r}$ today and at all future dates. These five values can be calculated from the five equations for values given in the definition of the research/obstruction equilibrium above (using $r=\tilde{r}$ and $\left(\pi_{1}, \pi_{2}\right)$ in those equations). Problem $\mathcal{P}$ then amounts to the following maximization problem:

$$
\max _{r, s} q_{L}(r) \tilde{v}_{2, L}+q_{F}(r, s) \tilde{v}_{2, F}+q_{M}(r, s) \tilde{v}_{1, L}+q_{B} \tilde{v}_{0}
$$

subject to $r+s=1$.

It is straightforward to show that under assumption (12), $\mathcal{P}$ is a concave problem. Suppose that we let $\omega$ denote the multiplier on the time constraint for the programming problem stemming from $\mathcal{P}$ (that is, $\omega$ is the shadow value of time). Then a necessary condition for a solution to $\mathcal{P}$ is that the marginal return to another unit of time spent in research equal the marginal return to another unit of time spent in obstruction (and both be equal to $\omega$ ) or 
that

$$
\begin{aligned}
& \frac{\partial q_{L}(r)}{\partial r} \cdot \tilde{v}_{2, L}+\frac{\partial q_{F}(r, s)}{\partial r} \cdot \tilde{v}_{2, F}+\frac{\partial q_{M}(r, s)}{\partial r} \cdot \tilde{v}_{1, L} \\
& =\frac{\partial q_{F}(r, s)}{\partial s} \cdot \tilde{v}_{2, F}+\frac{\partial q_{M}(r, s)}{\partial s} \cdot \tilde{v}_{1, L}=\omega
\end{aligned}
$$

where $\frac{\partial q_{L}(r)}{\partial r}$ is the derivative of $q_{L}$ with respect to $r, \frac{\partial q_{M}(r, s)}{\partial s}$ is the derivative of $q_{M}$ with respect to $s$, and so on. Research activity "takes" probability mass from the "events" F and $M$ (since $\frac{\partial q_{F}(r, s)}{\partial r}<0$ and $\left.\frac{\partial q_{M}(r, s)}{\partial r}<0\right)$ and "moves" it to the event $L$ (since $\frac{\partial q_{L}(r)}{\partial r}>0$ ). Obstruction takes probability mass from $F$ (since $\left.\frac{\partial q_{F}(r, s)}{\partial s}<0\right)$ and moves it to $M$ (since $\left.\frac{\partial q_{M}(r, s)}{\partial s}>0\right)$

The necessary condition above can be written as follows. Using $\frac{\partial q_{M}(r, s)}{\partial r}=-\frac{\partial q_{L}(r)}{\partial r}$ $-\frac{\partial q_{F}(r, s)}{\partial r}$ and $\frac{\partial q_{M}(r, s)}{\partial s}=-\frac{\partial q_{F}(r, s)}{\partial s}$, we can write the condition as

$$
\frac{\partial q_{L}(r)}{\partial r}\left[\tilde{v}_{2, L}-\tilde{v}_{1, L}\right]-\frac{\partial q_{F}(r, s)}{\partial r}\left[\tilde{v}_{1, L}-\tilde{v}_{2, F}\right]+\frac{\partial q_{F}(r, s)}{\partial s}\left[\tilde{v}_{1, L}-\tilde{v}_{2, F}\right]=0
$$

Given $\pi_{2} \geq \pi_{1}$, it is straightforward to show that $\tilde{v}_{2, L} \geq \tilde{v}_{1, L}$. It is also obvious that $\tilde{v}_{1, L} \geq$ $\tilde{v}_{2, F}$. So the first two terms in (14) are positive, and the last is negative. Next, note that $\frac{\partial q_{L}(r)}{\partial r}=(1-\delta) f^{\prime}(r), \frac{\partial q_{F}(r, s)}{\partial r}=-(1-\delta) f(1)(1-g(s)) f^{\prime}(r)$, and $\frac{\partial q_{F}(r, s)}{\partial s}=-(1-\delta) f(1)(1-$ $f(r)) g^{\prime}(s)$. Since $\lim _{r \rightarrow 0} f^{\prime}(r)=\infty$, the left-hand side of (14) is positive for $r$ close to zero. Since $\lim _{r \rightarrow 1} g^{\prime}(1-r)=\infty$, the left-hand side of (14) is negative for $r$ close to one. Hence, the unique solution to problem $\mathcal{P}$ (guaranteed by concavity) is an interior solution (guaranteed by the Inada-type conditions).

Let $\hat{r}(\tilde{r})$ be the unique solution to problem $\mathcal{P}$. The appendix shows that if $\hat{r}(\tilde{r})>\tilde{r}$, then $\hat{r}(\tilde{r})$ is strictly decreasing in $\tilde{r}$, and if $\hat{r}(\tilde{r})<\tilde{r}$, then $\hat{r}(\tilde{r})$ is strictly increasing in $\tilde{r}$. This is illustrated in Figure 1. Hence, there is a unique intersection point with the 45-degree line. Let $r^{*}$ be this unique intersection point. 
The point $r^{*}$ has the following property. Given that $r=r^{*}$ is pursued in all future periods, it is optimal to pursue this policy today. Hence, $r^{*}$ (and the corresponding values) satisfies the definition of symmetric, Markov-perfect equilibrium given above. Moreover, it is clear that it is the unique such point because any such point would have to satisfy $r=\hat{r}(r)$, and this is the only point to do so. Hence, the research/obstruction equilibrium exists and is unique.

Now, we turn to properties of the research/obstruction equilibrium research choice. Suppose that $\pi_{1}$ and $\pi_{2}$ are multiplied by some positive factor. It is straightforward to show that the values $\tilde{v}_{a, z}$ are multiplied by the same factor. Also, the curve $\hat{r}(\tilde{r})$ in Figure 1 is left unchanged by this multiplication. Hence, the solution to $r=\hat{r}(r)$ does not change. (Note that this would not be true if the time entrepreneurs could devote to these two activities were not fixed at one). Hence, the equilibrium $r^{*}$ depends on profits only through the ratio $\pi=\frac{\pi_{2}}{\pi_{1}}$. The appendix shows that if $\pi$ increases, the function $\hat{r}(\tilde{r})$ shifts up as illustrated in Figure 1. This shows that the equilibrium $r^{*}$ is strictly increasing in $\pi$.

\section{B. The Product-Market Equilibrium}

We start this subsection with a research level $r$ and solve for the resulting product-market equilibrium, calculating from this product-market equilibrium the profit ratio $\pi=\frac{\pi_{2}}{\pi_{1}}$. This derivation proceeds in two steps. First, given $r$, we solve for the stationary distribution of ladder rungs $\mu=\left(\mu_{0}, \mu_{1}, \mu_{2}\right)$. Then, given $\mu$, we solve for the equilibrium prices and quantities and the ratio of profits $\pi$. 


\section{The Derivation of Stationary Rung Proportions}

Suppose that $\mu_{0}$ industries are in the immature phase $a=0$ today. In stationary equilibrium, the following must be true:

$$
\mu_{0}=\left(1-2 q_{1}\right) \mu_{0}+\delta\left(1-\mu_{0}\right)
$$

where $q_{1}$ is the probability that a given entrepreneur advances to leadership at $a=1$ and is given in (10) above. The first term on the right-hand side is those industries that are at $a=0$ and remain. (With probability $2 q_{1}$, one of the two entrepreneurs advances to leadership at $a=1$; with probability $\left(1-2 q_{1}\right)$, neither advances.) The second term is those industries that are at $a \geq 1$ and fall to the bottom of the ladder (with probability $\delta$ ). Hence, the above equation can be solved for the stationary level of $\mu_{0}$,

$$
\mu_{0}=\frac{\delta}{2 q_{1}+\delta}
$$

Suppose that $\mu_{1}$ industries are at $a=1$ today. If each leader at $a=1$ engages in research $r$, then the following must be true in stationary equilibrium:

$$
\mu_{1}=q_{M}(r) \mu_{1}+\left[2 q_{1}\right] \mu_{0}
$$

The first term on the right-hand side is those industries that are at $a=1$ and remain. (A fraction $q_{M}(r)$ remain.) The second term is those industries in the immature phase that advance to $a=1$. (A fraction $2 q_{1}$ advance.) Hence, the above equation can be solved for the stationary level of $\mu_{1}$,

$$
\mu_{1}=\frac{2 q_{1} \mu_{0}}{1-q_{M}(r)}
$$

Given $\mu_{0}$ and $\mu_{1}$ from (15) and (16), we can solve for $\mu_{2}$ from $\mu_{0}+\mu_{1}+\mu_{2}=1$. 


\section{Current Period Profits}

Given the vector of rung proportions $\mu$, we can solve for the product-market equilibrium and then calculate the leader profits at each rung in that equilibrium. In order to discuss the product-market equilibrium, we must solve the utility maximization problem of households and the (static) profit maximization problem of entrepreneurs. Finally, we must consider market clearing conditions. Let us discuss each of these in turn.

\section{The Household Problem}

A representative household at each location earns a unit of income from the sale of its household time. In allocating its income to maximize utility, the household solves a three-stage budgeting procedure. First, it allocates income between manufactured goods and services. In the second stage, it divides the income allocated to manufactured goods among the local manufactured good and a composite of the foreign manufactured goods. In the third stage, given the income allocated to foreign manufactured goods, the household solves for the optimal mix of foreign goods. Here we solve these problems, starting from the third problem and working backward.

The third stage budgeting problem is to construct a unit of the foreign composite at the cheapest cost. Households at different locations will choose the same basket of foreign goods when making a basket to "produce" a unit of the foreign composite at the cheapest cost. $^{8}$ Hence, in solving this third stage budgeting problem, we need not keep track of location.

We assume for now that the price of a foreign good depends only on the ladder rung

\footnotetext{
${ }^{8} \mathrm{~A}$ household at location $j$ will obviously not use the manufactured good at $j$ in the foreign basket, while a household at location $i$ will put the manufactured good at $j$ in the foreign basket. The same is true for the manufactured good at $i$. (The household at $j$ will use the manufactured good at $i$ in the basket, but the household at $i$ will not.) But we can ignore this difference in baskets between households $i$ and $j$ because households are purchasing a continuum of goods.
} 
position in that country, that is, on $a$. (We will show below that this is true in equilibrium.) Let the foreign or export price be denoted $p_{E, a}$. Recall that the household faces a tariff on foreign goods, so the full price to a household of a unit purchase from an $a$-country is $(1+\tau) p_{E, a}$. Let $\tilde{b}_{a}$ denote the amount of good purchased from each $a$-country when the household minimizes the cost of constructing one unit of the foreign composite. The vector $\left(\tilde{b}_{0}, \tilde{b}_{1}, \tilde{b}_{2}\right)$ solves

$$
\min _{b_{0}, b_{1}, b_{2}} \sum_{a=0}^{2} \mu_{a}(1+\tau) p_{E, a} b_{a}
$$

subject to

$$
\left(\sum_{a=0}^{2} \mu_{a} b_{a}^{\frac{\sigma-1}{\sigma}}\right)^{\frac{\sigma}{\sigma-1}}=1
$$

and the solution is

$$
\widetilde{b}_{a}=\frac{\left(\frac{(1+\tau) p_{E, a}}{(1+\tau) p_{E, 0}}\right)^{-\sigma}}{\left[\sum_{a^{\prime}=0}^{A} \mu_{a^{\prime}}\left(\frac{(1+\tau) p_{E, a^{\prime}}}{(1+\tau) p_{E, 0}}\right)^{-(\sigma-1)}\right]^{\frac{\sigma}{\sigma-1}}} .
$$

The price of the foreign composite is then $\widetilde{p}_{C}=\sum_{a=0}^{A} \mu_{a}(1+\tau) p_{E, a} \widetilde{b}_{a}$, or

$$
\widetilde{p}_{C}=\left[\sum_{a=0}^{A} \mu_{a}\left((1+\tau) p_{E, a}\right)^{-(\sigma-1)}\right]^{-\frac{1}{\sigma-1}} .
$$

The second stage budgeting problem is to combine units of the foreign composite and the local manufactured good to produce a unit of the aggregate manufactured good at the cheapest cost. Suppose that a household is located in an $a$-country. We assume for now that the price of the domestic manufactured good depends only on the ladder rung position in the country, that is, on $a$. (We will show below that this is true in equilibrium.) The price of the domestic good faced by the household is denoted $p_{D, a}$ (which depends on $a$ ) and $p_{C}$ (which does not depend on $a$ ). Consider the problem of minimizing the cost of constructing one unit 
of the aggregate manufactured composite at a location $a$. Let $\left(\tilde{b}_{C, a}, \tilde{b}_{D, a}\right)$ be the solution. It solves

$$
\min _{b_{C, a}, b_{D, a}} \widetilde{p}_{C} b_{C, a}+p_{D, a} b_{D, a}
$$

subject to

$$
\left[(1-\lambda) b_{C, a}^{\frac{\sigma-1}{\sigma}}+\lambda b_{D, a}^{\frac{\sigma-1}{\sigma}}\right]^{\frac{\sigma}{\sigma-1}}=1
$$

and the solution is

$$
\begin{aligned}
& \widetilde{b}_{D, a}=\left[(1-\lambda)\left[\frac{(1-\lambda) p_{D, a}}{\lambda \widetilde{p}_{C}}\right]^{\sigma-1}+\lambda\right]^{-\frac{\sigma}{\sigma-1}} \\
& \widetilde{b}_{C, a}=\widetilde{b}_{D, a}\left[\frac{(1-\lambda) p_{D, a}}{\lambda \widetilde{p}_{C}}\right]^{\sigma} .
\end{aligned}
$$

The price of the aggregate manufactured good at $a$ is then the minimum cost,

$$
\widetilde{p}_{x, a}=\widetilde{p}_{C} \widetilde{b}_{C, a}+p_{D, a} \widetilde{b}_{D, a}=\left[(1-\lambda)^{\sigma}\left(\widetilde{p}_{C}\right)^{-(\sigma-1)}+\lambda^{\sigma}\left(p_{D, a}\right)^{-(\sigma-1)}\right]^{-\frac{1}{\sigma-1}} .
$$

The household maximization problem can now be expressed as choosing $x_{a}$ and $y_{a}$ to maximize $u(x, y)$ given in (1), subject to $\widetilde{p}_{x, a} x_{a}+y_{a} \leq 1$, noting that household income is one and that the price of services is $p_{y}=1$. This yields

$$
\widetilde{y}_{a}=\frac{1}{\left[(1-\alpha)^{\eta} \alpha^{-\eta} \tilde{p}_{x, a}^{1-\eta}+p_{y}\right]}
$$

and

$$
\widetilde{x}_{a}=\frac{1}{\left[\alpha^{\eta}(1-\alpha)^{-\eta} \widetilde{p}_{x, a}^{\eta}+\widetilde{p}_{x, a}\right]} .
$$




\section{The Profit Maximizing Prices}

The two entrepreneurs in each industry compete in prices in a Bertrand fashion. If the industry is in the immature phase, that is, $a=0$, then Bertrand competition yields price equal to marginal cost in both the local and the export market, so

$$
p_{D, 0}=p_{E, 0}=1 \text {. }
$$

Now consider the case where the industry is at $a=1$ or $a=2$. We first discuss what happens in the export market, then the domestic market.

In the leader's export market, export demand has a constant elasticity of demand equal to $\sigma$ (for prices less than the marginal cost of the follower). To see this, suppose that a leader in an $a$-country sets an export price $p_{E}^{\prime}$ and that the export price set by the leader in all other $a$-countries is $p_{E, a}$. Then if $x_{E}^{\prime}$ is the exports to a particular location of the leader setting price $p_{E}^{\prime}$ and $x_{E, a}$ is the exports of another $a$-country leader to that location, households at the location have a marginal rate of substitution condition that satisfies

$$
\left[\frac{x_{E}^{\prime}}{x_{E, a}}\right]^{-\frac{1}{\sigma}}=\frac{(1+\tau) p_{E}^{\prime}}{(1+\tau) p_{E, a}}
$$

or

$$
x_{E}^{\prime}=x_{E, a}\left[\frac{(1+\tau) p_{E}^{\prime}}{(1+\tau) p_{E, a}}\right]^{-\sigma} .
$$

If the leader setting $p_{E}^{\prime}$ adjusts this price, this adjustment has no effect on $x_{E, a}$ since the leader is measure zero. Hence, the export demand has a constant elasticity $\sigma$.

If there is no follower, the leader sets the markup over marginal cost equal to the inverse elasticity. Since marginal cost depends only on $a$ and the elasticity of export demand 
is the same at all locations, the profit maximizing price (when we ignore the follower) depends only on $a$. If we denote the price by $p_{E, a}$, it satisfies

$$
\frac{p_{E, a}-\frac{1}{\gamma^{a}}}{p_{E, a}}=\frac{1}{\sigma}
$$

or

$$
p_{E, a}=\frac{\sigma}{\sigma-1} \frac{1}{\gamma^{a}}
$$

But the leader cannot set the price higher than the follower's marginal cost, namely, $\frac{1}{\gamma^{a-1}}$. Hence, the equilibrium export price is the minimum of the price when we use the inverse elasticity rule and the follower's cost (which, again, depends only on $a$ ), that is,

$$
p_{E, a}=\min \left\{\frac{\sigma}{\sigma-1} \frac{1}{\gamma^{a}}, \frac{1}{\gamma^{a-1}}\right\}, a=1,2
$$

Using (24), the fact that $p_{E, 0}=1$, and the vector of rung proportions $\mu$ in the formula for $\widetilde{p}_{C}$, we can calculate the price of a unit of the foreign composite in the product-market equilibrium. Let us denote the price by $p_{C}^{* *}$. Since $\mu$ depends upon $r$, we can write the price of the foreign composite as a function of the research level $r$, namely, $p_{C}^{* *}(r)$. (Note, though, that the export prices $p_{E, a}$ do not depend upon $r$.)

Now consider the leader's domestic market. Domestic demand depends on the local price $p_{D}$, the price of the service good (which is, again, numeraire), and the price of the foreign composite $\widetilde{p}_{C}$. Since $\widetilde{p}_{C}$ does not depend on rung $a$, for notational simplicity, we write the demand function for the domestic manufactured good at a location as a function of $p_{D}$ alone, namely, $x_{D}\left(p_{D}\right)$. In order to analyze the leader's pricing strategy in the domestic market, we need to calculate the (own price) elasticity for domestic demand, which we denote 
$\varepsilon_{D}\left(p_{D}\right)$. The appendix shows that this elasticity is

$$
\varepsilon_{D}\left(p_{D}\right)=\varepsilon_{x}\left(p_{x}\right) \frac{p_{D} x_{D}\left(p_{D}\right)}{\widetilde{p}_{C} x_{C}\left(p_{D}\right)+p_{D} x_{D}\left(p_{D}\right)}+\sigma \frac{\widetilde{p}_{C} x_{C}\left(p_{D}\right)}{\widetilde{p}_{C} x_{C}\left(p_{D}\right)+p_{D} x_{D}\left(p_{D}\right)}
$$

where $x_{C}\left(p_{D}\right)$ denotes the demand for the foreign composite and $\varepsilon_{x}\left(p_{x}\right)$ is the (own price) elasticity of demand for the manufactured good $x$, which is given by

$$
\varepsilon_{x}\left(p_{x}\right)=\frac{\eta \alpha^{\eta}(1-\alpha)^{-\eta} p_{x}^{\eta}+p_{x}}{\alpha^{\eta}(1-\alpha)^{-\eta} p_{x}^{\eta}+p_{x}}
$$

and where, of course, $p_{x}$ is a function of $p_{D}$. Formula (25) states that the elasticity of demand for the domestic manufactured good is a weighted average of the elasticity of demand for $x$ and the elasticity of demand for exports (where the weights are spending on the domestic manufactured good as a percentage of total spending on manufactured goods and spending on the foreign composite as a percentage of total spending on manufactured goods, respectively).

Two results are key to analyzing the determination of the domestic price. First,

$$
\varepsilon_{D}\left(p_{D}\right)<\sigma
$$

To see this, recall our assumption that $\sigma>\eta$. Suppose first that $\eta \geq 1$. Then, examining (26), one can see that $\varepsilon_{x}<\eta<\sigma$. Hence, $\varepsilon_{D}\left(p_{D}\right)<\sigma$. If $\eta<1$, then $\varepsilon_{x}<1<\sigma$ (since we assume that $\sigma>1)$. Hence, $\varepsilon_{D}\left(p_{D}\right)<\sigma$. The second result is that

$$
\varepsilon_{D}\left(p_{D}\right) \text { is strictly increasing in } p_{D} \text {. }
$$

This is a consequence of two facts. First, as $p_{D}$ increases, the expenditure share on domestic manufactured goods declines. This places more weight on $\sigma$ and less on $\varepsilon_{x}$ in the formula (25). Second, it can be shown that $\varepsilon_{x}$ is nondecreasing in $p_{D}$. 
It is now straightforward to determine the profit maximizing domestic price, which we denote $p_{D, a}$. Suppose that the leader is at $a=1$ or $a=2$. Suppose that the following is true:

$$
\frac{\varepsilon_{D}\left(\frac{1}{\gamma^{a-1}}\right)}{\varepsilon_{D}\left(\frac{1}{\gamma^{a-1}}\right)-1} \frac{1}{\gamma^{a}} \geq \frac{1}{\gamma^{a-1}}
$$

Then the price that solves the markup rule from the first-order condition (given in (23)) exceeds the follower's marginal cost $\frac{1}{\gamma^{a-1}}$. In this case (where domestic demand is relatively inelastic), the leader sets price equal to the limit price, so $p_{D, a}=\frac{1}{\gamma^{a-1}}$. If (27) does not hold, then there exists a unique interior solution where the price solves the markup rule from the first-order condition. Hence, $p_{D, a}$ satisfies

$$
p_{D, a}=\min \left\{\frac{\varepsilon_{D}\left(p_{D, a}\right)}{\varepsilon_{D}\left(p_{D, a}\right)-1} \frac{1}{\gamma^{a}}, \frac{1}{\gamma^{a-1}}\right\}, a=1,2 .
$$

Note that since the domestic demand is less elastic than the export demand, the domestic price $p_{D, a}$ will be strictly greater than the export price $p_{E, a}$ unless the export price equals the limit price, in which case the export and the domestic prices both equal the limit price.

\section{Output and Profit Levels}

Given the profit maximizing prices determined above, we can calculate output and profit levels of a leader at any location. These levels depend only on the ladder rung $a$ in the leader's location.

First consider output levels. The leader sells at home and abroad. Domestic sales are calculated from the demand function for the domestic manufactured good, $x_{D, a}\left(p_{D, a}\right)$. In order to calculate the foreign sales of a leader in an a-country, let us first calculate the total world demand for the foreign composite. The world demand for the foreign composite is found by summing demand over all the locations, namely,

$$
x_{C, \text { world }}=\sum_{a^{\prime}=0}^{2} \mu_{a^{\prime}} x_{C, a^{\prime}}
$$


where $x_{C, a^{\prime}}$ is the demand for the foreign composite in an $a^{\prime}$-country, and there are $\mu_{a^{\prime}}$ such countries. A household, irrespective of location, will purchase $\tilde{b}_{a}$ units of the manufactured good from a leader in an $a$-country when constructing one unit of the foreign composite. Hence, the leader in an a-country can expect the following units in foreign sales or exports:

$$
x_{E, a}=\tilde{b}_{a} x_{C, w o r l d}
$$

The profit of the leader at $a$ is then

$$
\pi_{a}=\frac{p_{D, a}-\frac{1}{\gamma^{a}}}{p_{D, a}} p_{D, a} x_{D, a}+\frac{p_{E, a}-\frac{1}{\gamma^{a}}}{p_{E, a}} p_{E, a} x_{E, a}
$$

where profit is the sum of the profit in each market and where in each market, profit is a percentage-multiple of revenue. Given $\pi_{1}$ and $\pi_{2}$ from this formula, we can calculate the profit ratio, $\pi=\frac{\pi_{2}}{\pi_{1}} \cdot 9$

Recall that in the analysis of the research/obstruction game, we assumed that $\pi_{1} \geq 0$ and $\pi_{2} \geq \pi_{1}$ (condition (11)). Under what conditions will this be true? It is straightforward to show that if demand is price elastic in a market (either the market for the domestic manufactured good or the export market), then the profit in that market at $a=2$ is greater than at $a=1 .{ }^{10}$ If this is true in each market, then this must be true for the sum of profits

\footnotetext{
${ }^{9}$ Thus far, we have not discussed the issue of market-clearing. The key issue here is to make sure that the leader's demand for household time to produce manufactured goods does not exceed the unit of time available at the location. Otherwise, we could not focus on the interior equilibrium that has implicitly been assumed. Let the units of time demanded by a leader at $a$ be denoted $L_{a}$. The condition that demand not exceed one can be written as

$$
L_{a}=\left[x_{D, a}+x_{E, a}\right] \frac{1}{\gamma^{a}} \leq 1
$$

where $x_{D, a}$ and $x_{E, a}$ are the outputs produced by the leader that were calculated in the text. For the special case of $\eta=1$ (that is, Cobb-Douglass demand for the final goods $x$ and $y$ ), it is straightforward to show that $L_{a}$ is proportionate to $(1-\alpha)$. Hence, the condition holds if $\alpha$ is close enough to one.

${ }^{10}$ Let $p_{1}^{*}$ be the profit maximizing price in a market (either domestic or export) at $a=1$. An entrepreneur at $a=2$ can set $p_{2}=\frac{1}{\gamma} p_{1}^{*}$. (Note that this price is necessarily less than or equal to the follower's marginal
} 
in both markets. Now, export demand is price elastic. A sufficient condition for domestic demand to be price elastic is that $\eta \geq 1$. (See formula (25), and note that $\varepsilon_{x} \geq 1$ when $\eta \geq 1$.)

The analysis of this subsection began with a given $r$. From this $r$, we determined the implied stationary rung distribution $\mu$. From $\mu$, we calculated the product-market equilibrium and the implied profit ratio $\pi=\frac{\pi_{2}}{\pi_{1}}$. Let $\pi^{* *}(r)$ be the function that gives the profit ratio from the product-market equilibrium given $r$.

\section{Existence of Equilibrium}

In the analysis of the research/obstruction game, we determined the equilibrium amount of research given the profit ratio $\pi$, namely, $r^{*}(\pi)$. We plot $r^{*}(\pi)$ in Figure 2 . The function $r^{*}(\pi)$ is strictly increasing on the range $[1, \infty)$ and is bounded between zero and one. We also plot $\pi^{* *}(r)$, which is bounded and is greater than one for all $r \in[0,1]$. As we mentioned, a multicountry equilibrium corresponds to a pair $\left(\pi^{e}, r^{e}\right)$ such that $r^{e}=r^{*}\left(\pi^{e}\right)$ and $\pi^{e}=\pi^{* *}\left(r^{e}\right)$. In graphical terms, a multi-country equilibrium corresponds to a point in Figure 2 where the $r^{*}$ and $\pi^{* *}$ curves intersect. Since $r^{*}(\pi)$ is bounded between zero and one and is strictly increasing on the range $[1, \infty)$ and since $\pi^{* *}(r)$ is bounded and is greater than one for all $r \in[0,1]$, it is immediate that there exists a multi-country equilibrium.

We have also considered the issue of uniqueness. Figure 2 is a graph of $r^{*}$ and $\pi^{* *}$ for actual parameter values. For these values, the intersection and, hence, the multi-country equilibrium are unique. In Figure 2, the $\pi^{* *}$ function is nearly a straight line. The profit

cost.) The entrepreneur's profit at $a=2$ is then

$$
\frac{\frac{1}{\gamma} p_{1}^{*}-\frac{1}{\gamma^{2}}}{\frac{1}{\gamma} p_{1}^{*}} p_{2} x_{2}=\frac{p_{1}^{*}-\frac{1}{\gamma}}{p_{1}^{*}} p_{2} x_{2} .
$$

This is larger than the profit at $a=1$ because revenues are higher since demand is elastic. 
ratio $\pi^{* *}$ does not vary much with $r$. To the extent that the $\pi^{* *}$ function looks like a straight line relative to the $r^{*}$ function, the equilibrium will obviously be unique. The $\pi^{* *}$ function is quite complicated, and an analytical proof of uniqueness appears to be beyond our grasp. We have used the computer to calculate the $r^{*}$ and $\pi^{* *}$ functions for a wide range of parameters. For all the parameter sets that we considered, the equilibrium is unique. For the remaining analysis of the paper, we assume that the $r^{*}$ and $\pi^{* *}$ functions have a unique intersection as they do for the parameters used to construct Figure 2.

\section{Lower Tariffs: More Research, Less Obstruction}

This section presents the main result of the paper. In particular, we compare the research undertaken in a multi-country equilibrium when each country has tariff $\tau_{H}$ to that undertaken when each country has tariff $\tau_{L}, \tau_{L}<\tau_{H}$. We show that the leader in each country does more research (when $a=1$ ) when the tariff is $\tau_{L}$. Hence, the stationary distribution of rung proportions $\mu$ is higher when the tariff is $\tau_{L}$.

We use Figure 3 to begin our analysis of comparing multi-country equilibria under different tariffs. A change in the tariff has no effect on the $r^{*}$ curve. However, the $\pi^{* *}$ curve depends on $\tau$. Write the $\pi^{* *}$ function as $\pi^{* *}(r, \tau)$ to denote its dependence on $\tau$. In the proposition below, we show that $\pi^{* *}$ is decreasing in $\tau$. Hence, a decrease in tariffs shifts the $\pi^{* *}$ curve to the right in Figure 3. This means research $r^{e}$ is higher in the multi-country equilibrium with the lower tariff.

With this introduction, we turn to the main proposition. Before stating the proposition, let us make the following assumptions:

$$
\sigma \leq \frac{\gamma}{\gamma-1}
$$


and

$$
\eta \geq 4-3 \sigma .
$$

Then we have the following proposition.

Proposition. Assume that the parameter conditions (4), (30), and (31) hold. Then $\pi^{* *}(r, \tau)$ is strictly decreasing in $\tau$. Hence, in multi-country equilibria, the lower are tariffs, the higher is research and the lower is obstruction.

We offer a few comments about this proposition: First, obviously and importantly, we note that the distribution of $\mu$ shifts up with lower tariffs. Lower tariffs lead to better techniques for two reasons: more research and less invalidation of follower successes. Second, the restriction on $\eta$ is mild since we are already assuming that $\sigma>1$. For example, condition (31) will necessarily hold if $\eta \geq 1$ or if $\sigma>\frac{4}{3}$. Third, the intuition for the proposition, described shortly, indicates that large $\sigma$ makes the result more likely to hold. We restrict $\sigma$ to be small (as in condition (30)) since this leads to a considerable technical simplification by insuring that the prices (both domestic and foreign) equal the limit price and so do not change with $\tau$. Below, we look at a numerical example to show that lowering tariffs has the same effects when $\sigma$ is large.

The proof of the proposition is in the appendix. Here, we sketch a proof for the case where $\eta=1$ and $\gamma$ is close to 1 . This sketch provides much of the intuition for the result.

Recall the expression for profit at ladder rung a given in (29), namely,

$$
\pi_{a}=\frac{p_{D, a}-\frac{1}{\gamma^{a}}}{p_{D, a}} p_{D, a} x_{D, a}+\frac{p_{E, a}-\frac{1}{\gamma^{a}}}{p_{E, a}} p_{E, a} x_{E, a}
$$


which gives profit as the sum of profit in each market, where in each market, profit is a percentage-multiple of revenue. Recall that condition (30) implies that the domestic and export prices are equal. Hence, at a given $a$, the percentage-multiples is the same in both markets. Moreover, since prices satisfy $p_{D, 1}=p_{E, 1}=p_{1}=1$ and $p_{D, 2}=p_{E, 2}=p_{2}=\frac{1}{\gamma}$, the percentage-multiples at $a=1$ and $a=2$ both equal $\left(1-\frac{1}{\gamma}\right)$. Hence, the ratio of profits can be expressed as the ratio of revenues

$$
\pi=\frac{p_{2} x_{D, 2}+p_{2} x_{E, 2}}{p_{1} x_{D, 1}+p_{1} x_{E, 1}}
$$

The ratio of profits can further be written as

$$
\pi=k_{D, 1} \frac{p_{2} x_{D, 2}}{p_{1} x_{D, 1}}+\left(1-k_{D, 1}\right) \frac{p_{2} x_{E, 2}}{p_{1} x_{E, 1}}
$$

where $k_{D, 1}$ is defined as the share of domestic sales in total sales of a leader at $a=1$, that is,

$$
k_{D, 1}=\frac{p_{1} x_{D, 1}}{\left(p_{1} x_{D, 1}+p_{1} x_{E, 1}\right)} .
$$

Expression (32) gives the ratio of profits as a weighted sum of the ratio of revenues in the two markets. Recall that as a leader makes a step up the ladder from rung $a=1$ to rung $a=2$, the leader cuts the domestic and export price from $p_{1}=1$ to $p_{2}=\frac{1}{\gamma}$. Hence, the ratio of revenues in each market is related to the elasticity of demand in each market. Dropping the $D$ and $E$ subscripts, the ratio of revenues in a market can be expressed as

$$
\frac{p_{2} x_{2}}{p_{1} x_{1}} \simeq\left(\frac{1}{\gamma}-1\right)\left(1-\varepsilon\left(p_{1}\right)\right)+1
$$

where we have used $p_{1}=1$ and $p_{2}=\frac{1}{\gamma} \cdot{ }^{11}$ The approximation holds for $\gamma$ close to one. Note that the ratio of revenues is increasing in $\varepsilon\left(p_{1}\right)$. Hence, when we assume that the weight

\footnotetext{
${ }^{11}$ This formula can be derived as follows. Let $R(p)=p x(p)$ denote revenues. Then $R^{\prime}=x(p)(1-\varepsilon(p))$
} 
$k_{D, 1}$ does not depend on $\tau$, the question of how the ratio of profits depends on the tariff is equivalent to asking how the elasticity of demand in each market depends on $\tau$. In what follows, we write elasticities as $\varepsilon(p, \tau)$ to emphasize their dependence on $\tau$.

Let us first consider how the elasticity of demand for the local manufactured good depends on $\tau$. Recall the formula for the elasticity of demand for the local good, $\varepsilon_{D}\left(p_{D}, \tau\right)$, given in expression (25) above. The elasticity is a weighted average of $\varepsilon_{x}\left(p_{x}, \tau\right)$ and $\sigma$, where $\varepsilon_{x}\left(p_{x}, \tau\right)<\sigma$. Now compare the elasticity at $\tau_{L}$ and $\tau_{H}, \tau_{L}<\tau_{H}$. At the lower tariff, spending on foreign manufactured goods accounts for a larger share of total spending. Hence, greater weight is placed on $\sigma$. This effect leads $\varepsilon_{D}\left(p_{D}, \tau\right)$ to be higher. The only remaining question is how $\varepsilon_{x}\left(p_{x}, \tau\right)$ depends on the tariff. With $\eta=1, \varepsilon_{x}\left(p_{x}, \tau\right)=1$. Hence, demand for the local manufactured good is more elastic at the lower tariff.

We can also give this argument in words. Suppose that the tariff is extremely large so that, approximately, households purchase only the local manufactured good and services. Then as the leader cuts the domestic price, households make some substitution from services to the local manufactured good. The extent of substitution depends on $\eta$. If tariffs are low when a leader cuts the domestic price, households make substitutions from services and foreign manufactured goods to the local manufactured good. Since the foreign and local manufactured goods are better substitutes for each other (depending on $\sigma$ ) than are the local manufactured good and services, demand is more elastic.

The elasticity of demand for the leader's exports equals $\sigma$. Hence, the elasticity does

where $R^{\prime}$ is the derivative of revenues with respect to $p$. We also have the following approximation:

$$
R^{\prime} \simeq \frac{p_{2} x\left(p_{2}\right)-p_{1} x\left(p_{1}\right)}{p_{2}-p_{1}}
$$

where $p_{2}=\frac{1}{\gamma}$ and $p_{1}=1$. Using these expressions, we can derive the expression in the text. 
not depend on $\tau$. The ratio of foreign revenues therefore does not depend on $\tau$.

If the weight $k_{D, 1}$ did not depend on $\tau$, then the sketch of the proof would be done. However, the weight $k_{D, 1}$ does change with $\tau$. It can be shown (as in the appendix) that as tariffs are lowered, $p_{1} x_{E, 1}$ increases and $p_{1} x_{D, 1}$ decreases. Hence, domestic sales make up a smaller share of total sales at a lower tariff. (That is, $k_{D, 1}$ is smaller at the lower tariff.) Hence, at the lower tariff, more weight is placed on the ratio of foreign revenues. But since the elasticity of export demand exceeds the elasticity of demand for the local manufactured good (that is, $\sigma>\varepsilon_{D}\left(p_{D}, \tau\right)$ ), the ratio of foreign revenues exceeds the ratio of domestic revenues. Hence, the shifting weights also work to increase the profit ratio. This completes the proof that $\pi^{* *}(r, \tau)$ strictly decreases in $\tau$.

Before proceeding, we mention that it is possible to conduct another experiment: compare the research undertaken in a given country when each country (including itself) has tariff $\tau_{H}$ to that undertaken when the given country has tariff $\tau_{L}$ and all other countries have tariff $\tau_{H}$. The unilateral reduction in the tariff will have no effect on the leader's export revenues, that is, $p_{2} x_{E, 2}$ and $p_{1} x_{E, 1}$, so $p_{1} x_{E, 1}$ will be constant rather than increase as it did above. Still, the weight $k_{D, 1}$ will decrease in $\tau$. All the other arguments are the same. Hence, research is higher in the given country when it has tariff $\tau_{L}$.

\section{Some Numerical Results}

As mentioned above, the parameter restriction in (30), namely, $\sigma \leq \frac{\gamma}{\gamma-1}$, implies that the domestic and export prices equal the limit prices. This simplifies the analysis since the prices do not vary with $\tau$. For the case of $\sigma>\frac{\gamma}{\gamma-1}$, the export prices are necessarily interior (that is, below the limit prices), and the domestic prices may or may not be interior. If 
$\sigma>\frac{\gamma}{\gamma-1}$ and if the domestic prices $p_{D, 1}$ and $p_{D, 2}$ are equal to the limit prices, then the proof of the proposition in the appendix can be extended in a straightforward fashion. However, we have not been able to prove the proposition for the case where the domestic prices are interior. What makes the problem intractable is that as $\tau$ is varied, the interior profit maximizing domestic prices vary.

While the proposition is hard to prove for the case of large $\sigma$, we believe that it is for large $\sigma$ that the effects of a reduction in $\tau$ are actually the largest. In the proof of the proposition sketched above, the fact that $\sigma$ is large is used in a number of places to establish points. So, in closing this section, we show in a numerical example, which violates condition (30), that decreases in tariffs increase research. The numerical example also illustrates that the effect of tariff reductions on research effort can be large.

For this example, we assume that $\gamma=2$. The proposition therefore applies for $\sigma \leq$ $\frac{\gamma}{\gamma-1}=2$. We therefore consider a larger $\sigma, \sigma=5$. For the remaining preference parameters, we choose $\eta=1, \alpha=0.7, \lambda=0.5$, and $\beta=0.5$. For the remaining technology parameters, we choose $\delta=0.3$ for the probability of a return to an immature phase, while for the probability functions, we choose

$$
\begin{aligned}
& f(r)=.2 r^{.5} \\
& g(s)=.6 s^{.5} .
\end{aligned}
$$

In this example, the obstruction technology works well compared to the research technology. Specifically, the blocking technology is three times as likely to be successful for a given amount of time input as the research technology. For this specification of the probability functions, 
the concavity condition (12) holds.

Table 1 presents the multi-country equilibrium for various levels of the tariff $\tau$. Consider first the case where $\tau=\infty$. In this extreme case, the tariff is prohibitive, and there is no trade. In the unique equilibrium of the economy, the leader at $a=1$ allocates most of his or her time to obstruction, that is, $s=0.96$, spending only $r=0.04$ on research. In this case, the probability that the leader advances from $a=1$ to $a=2$ is only 0.04 . The probability of obstructing a successful follower is 0.59 . In this equilibrium, the fraction of countries at the highest productivity level is $\mu_{2}=0.1$.

Note that the profit ratio equals one when $\tau=\infty$; that is, the current profit $\pi_{2}$ at rung 2 equals the current profit $\pi_{1}$ at rung 1 . To see this, recall that $\eta=1$ for this example. This means that the household preferences for services and the local manufactured good are Cobb-Douglas. Hence, the elasticity of demand for the local manufactured good is one. Since the demand for the domestic manufactured good has unit elasticity, the leader can increase profit by increasing price. The leader raises the domestic price until it equals the limit price, that is, $p_{D, 1}=1, p_{D, 2}=0.5($ where $\gamma=2)$. We can write profit at $a$ as

$$
\pi_{a}=\frac{p_{D, a}-\frac{1}{\gamma^{a}}}{p_{D, a}} p_{D, a} x_{D, a} .
$$

The first term, the percentage-multiple, does not depend on $a$. The percentage-multiple is $\left(1-\frac{1}{\gamma}\right)=0.5$. This means the profit of the leader equals one-half the revenues at each rung. Since preferences are Cobb-Douglas, total spending on the local manufactured good is a fixed fraction $(1-\alpha)=0.3$ of the household's income. Hence, revenue, as well as profit, at $a=1$ is the same as that at $a=2$.

Now consider the case where $\tau$ is less than infinity. For such $\tau$, a leader has an export 
market as well as a domestic market. The export price set by a leader does not depend on $\tau$ since the elasticity of export demand is $\sigma=5$ regardless of the tariff. Hence, the export price at $a>0$, when we use the markup over marginal cost rule, is $p_{E, a}=\frac{\sigma}{\sigma-1} \frac{1}{\gamma^{a}}=1.25 / 2^{a}$, or $p_{E, 1}=0.63$ and $p_{E, 2}=0.31$. The domestic price does vary with $\tau$, however. There are three cases. For $\tau>1.85$, domestic demand is sufficiently elastic at both rungs $a=1$ and $a=2$ so that domestic prices equal the limit prices, that is, $p_{D, 1}=1, p_{D, 2}=\frac{1}{2}$. For $\tau$ between 0.45 and 1.85 , the domestic price at $a=1$ is below the limit price and at $a=2$ equals the limit price. For $\tau$ less than 0.45 , domestic demand is sufficiently elastic such that the equilibrium domestic prices at both rungs are below the limit prices.

The main point that Table 1 illustrates is that decreases in $\tau$ lead to increases in the profit ratio $\pi=\frac{\pi_{2}}{\pi_{1}}$. This in turn increases the amount of research and decreases the amount of obstruction. So, even though $\sigma$ violates condition (30), the results of the proposition hold. The quantitative effects of lowering tariffs in the example are fairly dramatic. The profit ratio goes from $\pi=1$ at $\tau=\infty$ to $\pi=10$ with free trade. In autarky, the amount of research is $r=0.04$, while with free trade, $r=0.99$. The fraction of countries at the top of the ladder increases from 0.10 with autarky to 0.21 with free trade.

\section{Discussion}

In constructing the above model, we made a number of simplifying assumptions. We now briefly discuss the reasons for the assumptions and the extent to which they can be relaxed. We also discuss some related literature.

\section{Remark 1.}

In the model, we assume a finite technology-ladder. This is different from, for example, 
Grossman and Helpman (1991), where there is no upper bound on the ladder. We do this for technical reasons. Grossman and Helpman (1991) assume log utility (that is, $\sigma=\eta=1$ ), which implies that the expenditure shares for each good are fixed. Hence, the revenue earned by a leader in a country is constant over time. This means that the profit earned by an entrepreneur in a country in a period depends only on his ladder position and that of his rival. This is a considerable simplification since the equilibrium research in each country can be determined without knowledge of the ladder positions of leaders in other countries (that is, the distribution $\mu$ ). In our analysis, it is essential that we use general CES preferences since we need the ratio of profits, as well as the level of revenues and profits, to change as tariffs are altered. (See Table 1.) With general CES preferences, equilibrium research depends on $\mu$. Given that we introduce this new dimension of general CES preferences, it helps to simplify the model along another dimension - the use of a finite ladder.

In the model, we assume that the ladder has only three rungs. We conjecture that the results would hold in a model with a general number of ladder rungs, say, $A$. We have two reasons to support this conjecture. First, we have studied the research/obstruction game with a general number of rungs. In place of condition (11), we assume that the ratio of profits between adjacent rungs is a constant, that is, $\pi_{a+1} / \pi_{a}=\pi \geq 1$, for $a \in\{0,1, \ldots, A-1\}$. In numerical examples that we have explored, the equilibrium research at rung $a$ in the research/obstruction game, say, $r_{a}^{*}(\pi)$, increases at each $a$ as the constant $\pi$ increases. Second, the logic for why a decrease in tariffs increases the ratio of profits between rung $a=2$ and rung $a=1$ in the above model may hold more generally, implying that a decrease in tariffs increases the ratio of profits between adjacent rungs on a more general ladder. 


\section{Remark 2.}

In the model, when a manufacturing industry is at $a=1$, the leader can engage in research or obstruction, but the follower engages only in research. Consider a more general model where the follower can engage in obstruction at $a=1$. If the follower is successful in obstruction in a given period, then the leader will not be permitted to advance to leadership at $a=2$ in the following period. Given the Inada-type conditions assumed for $g(s)$, the follower will engage in some obstruction. So, this change in the model requires additional analysis. However, we can show that a reduction in the tariff will have no effect on the follower's research and obstruction choice at $a=1$. Hence, a reduction in the tariff has the same effect as before: it increases the leader's research at $a=1$. Hence, our fundamental comparative statics result contained in the proposition will not change.

\section{Remark 3.}

We assume that the total time an entrepreneur devotes to ascending to and maintaining a technology leadership position is fixed at one. Our interest is in how this time is divided between the two activities, research and obstruction. This distinguishes our research on quality ladders from the previous literature where there is typically only a single activity that influences ladder position (that is, research), but where the time or resources devoted to this activity can vary. (See, for example, Grossman and Helpman (1991).) Suppose that we add this additional margin into the analysis; that is, suppose that we assume that the total time an entrepreneur can devote to ascending to and maintaining a technology leadership position can be varied. (For example, we can add a labor/leisure margin for the entrepreneurs.) Consider now what happens when tariffs are reduced. It is clear that a tariff reduction has an ambiguous effect on the amount of time devoted to ascending to and 
maintaining a technology leadership position. On the one hand, a reduction in the tariff means greater export profit is possible if an entrepreneur advances, so an entrepreneur may want to increase total time devoted to the activity. On the other hand, more competition from entrepreneurs at advanced ladder rung positions may decrease the returns to allocating time to the activity.

\section{Remark 4.}

We assume that the obstruction activity is to prevent the domestic rival from advancing. One can imagine a model where entrepreneurs can also lobby for higher tariffs on imports of goods; that is, they can try to obstruct foreign rivals as well. There will then be three uses of entrepreneurial time: research, obstruction of domestic rivals (through regulations), and obstruction of foreign rivals (through attempts to increase tariffs). This would be a complicated model. But imagine that we have solved for a division of time among the three activities. Our policy experiment in this world would be the following: What are the effects of adopting an institution (such as GATT or the World Trade Organization) which, for a given amount of effort, will make it less likely that the lobbying for higher tariffs will be successful? In such a model, while complicated to analyze, we expect adoption of such a pro-trade institution to have two effects. First, with the amount of time devoted to lobbying for higher tariffs held fixed, the institution will lead to a shift of the remaining time from obstruction to research (because of lower tariffs). Second, the institution may reduce time devoted to lobbying for tariffs since the likelihood of success is lower.

\section{Remark 5.}

There is an old debate in the industrial organization literature concerning what market structure, monopoly or competition, leads to greater industry productivity over time. As we men- 
tioned in the introduction, there is an old view, as expressed in Leibenstein (1966), that monopoly leads to inefficiency. ${ }^{12}$ But there is an opposite and even older wisdom, due to Schumpeter, that monopoly leads to higher productivity. The logic behind this latter wisdom is that in order to invest in research, an entrepreneur must be able to earn monopoly returns for some period if the research is successful. This logic has been formalized in, for example, Aghion and Howitt (1992) (AH). In that paper, AH examine what happens to equilibrium research as the elasticity of demand for the product of the current monopolist increases. AH interpret this increase in elasticity as an increase in competition. AH show that as competition increases (that is, the elasticity of demand) research and, hence, productivity fall.

It is interesting to perform AH's comparative static in our model. Consider the above model in the special case where $\lambda=0$ in (2). In this case, there is no domestic manufactured good. The model can be interpreted as a model of a single location. In this version of the model, AH's comparative statics exercise corresponds to increasing $\sigma$ in (3). The increase in $\sigma$ means the elasticity of demand for each product increases. What effect does this have in our model? In this version of the model, it can be shown that the ratio of profits is ${ }^{13}$

$$
\pi=\frac{\pi_{2}}{\pi_{1}}=\gamma^{\sigma}
$$

Hence, increases in $\sigma$ lead to increases in the profit ratio and, hence, to increases in research (and reductions in obstruction). So, while total research effort decreases in AH as the elasticity of demand increases, in our model, where the total time devoted to ascending to and

\footnotetext{
${ }^{12}$ The logic for this wisdom in early writings is often not straightforward. However, see the recent paper by Parente and Prescott (1997) for a formal model showing how monopoly can lead to very low productivity.

${ }^{13}$ The profit ratio in $\mathrm{AH}$ is identical to the profit ratio in this version of the model.
} 
maintaining a technology leadership position is fixed, increases in the elasticity of demand lead to a change in the composition of time devoted to this activity: research becomes a larger fraction of effort, obstruction a smaller fraction.

\section{Appendix}

\section{A. Results for Section 4.1}

This part of the appendix proves results that together imply the lemma stated in Section 4.1.

Lemma A1. If $\hat{r}(\tilde{r})>\tilde{r}$, then $\hat{r}(\tilde{r})$ is strictly decreasing in $\tilde{r}$, and if $\hat{r}(\tilde{r})<\tilde{r}$, then $\hat{r}(\tilde{r})$ is strictly increasing in $\tilde{r}$.

Proof. As discussed in the text, there is a unique interior solution $\hat{r}(\tilde{r})$ to problem $\mathcal{P}$ that solves the FONC (14). From inspection of the FONC (14), it is immediate that the claim is true if and only if the ratio

$$
\frac{\left[\tilde{v}_{2, L}-\tilde{v}_{1, L}\right]}{\left[\tilde{v}_{1, L}-\tilde{v}_{2, F}\right]}
$$

is strictly decreasing in $\tilde{r}$ for any $\tilde{r}$ satisfying $\hat{r}(\tilde{r})>\tilde{r}$ and strictly increasing in $\tilde{r}$ for any $\tilde{r}$ satisfying $\hat{r}(\tilde{r})<\tilde{r}$.

Case 1: $\hat{r}(\tilde{r})>\tilde{r}$. We show that the numerator of (33) is decreasing in $\tilde{r}$ and the denominator is increasing. This will prove that (33) is decreasing in $\tilde{r}$ for any $\tilde{r}$ satisfying $\hat{r}(\tilde{r})>\tilde{r}$.

We first show that the numerator is decreasing; that is,

$$
\frac{d \tilde{v}_{2, L}}{d \tilde{r}}<\frac{d \tilde{v}_{1, L}}{d \tilde{r}}
$$

To see this, note first that

$$
\tilde{v}_{2, L}=\pi_{2}+\beta\left[(1-\delta) \tilde{v}_{2, L}+\delta \tilde{v}_{0}\right]
$$


which implies that

$$
\tilde{v}_{2, L}=\frac{\pi_{2}}{1-\beta(1-\delta)}+\frac{\beta \delta}{1-\beta(1-\delta)} \tilde{v}_{0} .
$$

Analogously,

$$
\tilde{v}_{2, F}=\frac{\beta \delta}{1-\beta(1-\delta)} \tilde{v}_{0}
$$

This implies that

$$
\frac{d \tilde{v}_{2, L}}{d \tilde{r}}=\frac{d \tilde{v}_{2, F}}{d \tilde{r}}=\frac{\beta \delta}{1-\beta(1-\delta)} \frac{d \tilde{v}_{0}}{d \tilde{r}}
$$

We can rewrite (36) as

$$
\beta \delta \frac{d \tilde{v}_{0}}{d \tilde{r}}=[1-\beta(1-\delta)] \frac{d \tilde{v}_{2, L}}{d \tilde{r}}
$$

and this will be used below.

Next, note that we can write $\tilde{v}_{1, L}$ as

$$
\begin{aligned}
\tilde{v}_{1, L} & =\pi_{1}+\beta\left[q_{L}(\tilde{r}) \tilde{v}_{2, L}+q_{F}(\tilde{r}) \tilde{v}_{2, F}+q_{M}(\tilde{r}) \tilde{v}_{1, L}+q_{B} \tilde{v}_{0}\right] \\
& =\pi_{1}+\beta(1-\delta) \tilde{v}_{n o f a l l}+\beta \delta \tilde{v}_{0}
\end{aligned}
$$

where $\tilde{v}_{\text {nofall }}$ is the discounted payoff next period conditioned upon the fact that the industry does not fall off the ladder in the next period,

$$
\begin{aligned}
\tilde{v}_{\text {nofall }}= & f(\tilde{r}) \tilde{v}_{2, L}+f(1)[1-f(\tilde{r})][1-g(1-\tilde{r})] \tilde{v}_{2, F} \\
& +[1-f(\tilde{r})-f(1)[1-f(\tilde{r})][1-g(1-\tilde{r})]] \tilde{v}_{1, L} .
\end{aligned}
$$

The slope of $\tilde{v}_{\text {nofall }}$ is

$$
\frac{d \tilde{v}_{\text {nofall }}}{d \tilde{r}}=\mathcal{A}(\tilde{r})+\mathcal{B}(\tilde{r}) \frac{d \tilde{v}_{2, L}}{d \tilde{r}}+[1-\mathcal{B}(\tilde{r})] \frac{d \tilde{v}_{1, L}}{d \tilde{r}}
$$


where the function $\mathcal{A}(\tilde{r})$ is defined to be that part of the derivative of (39) that includes the effect of the change in $\tilde{r}$ on the probability terms $f(\tilde{r})$ and $g(1-\tilde{r})$ but leaves $\tilde{v}_{2, L}, \tilde{v}_{2, F}$, and $\tilde{v}_{1, L}$ fixed; that is,

$$
\begin{aligned}
\mathcal{A}(\tilde{r}) \equiv & f^{\prime}(\tilde{r})\left[\tilde{v}_{2, L}-\tilde{v}_{1, L}\right] \\
& +f^{\prime}(\tilde{r}) f(1)[1-g(1-\tilde{r})]\left[\tilde{v}_{1, L}-\tilde{v}_{2, F}\right] \\
& -g^{\prime}(1-\tilde{r}) f(1)[1-f(\tilde{r})]\left[\tilde{v}_{1, L}-\tilde{v}_{2, F}\right] .
\end{aligned}
$$

Further, the last two terms of (40) include the effects of a change in $\tilde{r}$ on $\tilde{v}_{2, L}, \tilde{v}_{2, F}$, and $\tilde{v}_{1, L}$. We also use $(36)$ to substitute $\frac{d \tilde{v}_{2, L}}{d \tilde{r}}$ for $\frac{d \tilde{v}_{2, F}}{d \tilde{r}}$. Finally, $\mathcal{B}(\tilde{r})$ is the probability that the incumbent or the entrant advances:

$$
\mathcal{B}(\tilde{r}) \equiv f(\tilde{r})+f(1)[1-f(\tilde{r})][1-g(1-\tilde{r})]
$$

We next claim that

$$
\mathcal{A}(\tilde{r})>0
$$

This follows from the fact that the objective function (13) is concave and the fact that $\tilde{r}$ is by assumption strictly less than the maximizer $\hat{r}_{1}(\tilde{r})$ of this function. (Compare (41) to (14).) Equations (38) and (40) and the fact that $\mathcal{A}(\tilde{r})>0$ imply that

$$
\begin{aligned}
\frac{d \tilde{v}_{1, I}}{d \tilde{r}} & =\beta(1-\delta) \frac{d \tilde{v}_{n o f a l l}}{d \tilde{r}}+\beta \delta \frac{d \tilde{v}_{0}}{d \tilde{r}} \\
& >\beta(1-\delta)\left[\mathcal{B}(\tilde{r}) \frac{d \tilde{v}_{2, L}}{d \tilde{r}}+[1-\mathcal{B}(\tilde{r})] \frac{d \tilde{v}_{1, L}}{d \tilde{r}}\right]+\beta \delta \frac{d \tilde{v}_{0}}{d \tilde{r}}
\end{aligned}
$$


Substituting (37) into the above yields

$$
\begin{aligned}
\frac{d \tilde{v}_{1, L}}{d \tilde{r}}> & \beta(1-\delta)\left[\mathcal{B}(\tilde{r}) \frac{d \tilde{v}_{2, L}}{d \tilde{r}}+[1-\mathcal{B}(\tilde{r})] \frac{d \tilde{v}_{1, L}}{d \tilde{r}}\right] \\
& +[1-\beta(1-\delta)] \frac{d \tilde{v}_{2, L}}{d \tilde{r}} \\
= & {[1-\beta(1-\delta)(1-\mathcal{B}(\tilde{r}))] \frac{d \tilde{v}_{2, L}}{d \tilde{r}} } \\
& +\beta(1-\delta)(1-\mathcal{B}(\tilde{r})) \frac{d \tilde{v}_{1, L}}{d \tilde{r}} .
\end{aligned}
$$

This directly implies the inequality (34) that we were required to show. This proves that the numerator is decreasing.

We next show that the denominator is increasing; that is,

$$
\frac{d \tilde{v}_{1, L}}{d \tilde{r}}>\frac{d \tilde{v}_{2, F}}{d \tilde{r}}
$$

This follows from (34) and (36). This concludes the proof for Case 1. Q.E.D.

Case 2: $\hat{r}(\tilde{r})<\tilde{r}$. The proof for this case follows the same arguments as for Case 1, except that $\mathcal{A}(\tilde{r})<0$ instead of $\mathcal{A}(\tilde{r})>0$. Q.E.D.

Lemma A2. Let $\hat{r}(\tilde{r}, \pi)$ solve the constrained problem in the text for a given $\tilde{r}$ and a given ratio of profits $\pi=\frac{\pi_{2}}{\pi_{1}}$. The solution $\hat{r}(\tilde{r}, \pi)$ is strictly increasing in the ratio $\pi$.

\section{Proof.}

It is easiest to prove this by fixing $\pi_{2}$ and varying $\pi_{1}$. Let $\hat{r}\left(\tilde{r}, \pi_{1}, \pi_{2}\right)$ be the solution for fixed levels of $\pi_{1}$ and $\pi_{2}$. We need to show that $\hat{r}_{1}$ is strictly decreasing in $\pi_{1}$ for fixed $\pi_{2}$. Following the proof of the above Lemma A1, we need to show that the ratio (33) is strictly decreasing in $\pi_{1}$. As before, we show this by proving that the numerator is decreasing while 
the denominator is increasing. Note first that

$$
\frac{\partial \tilde{v}_{2, L}}{\partial \pi_{1}}=\frac{\partial \tilde{v}_{2, F}}{\partial \pi_{1}}
$$

obviously holds since, if the leader is at $a=2$, the only way a change in $\pi_{1}$ will make a difference is if there is a fall off the ladder, and if that happens, it doesn't matter whether the firm was the leader or the follower while the rung was $a=2$. Next note that

$$
\frac{\partial \tilde{v}_{1, L}}{\partial \pi_{1}}>\frac{d \tilde{v}_{2, L}}{\partial \pi_{1}}
$$

obviously holds. Conditions (43) and (44) imply that the numerator is decreasing and that the denominator is increasing. Q.E.D.

\section{B. Result for Section 4.2.2}

Section 4.2.2 presents a formula for the own-price elasticity of demand for the domestic manufactured good. This part of the appendix derives this formula.

We start with a useful derivation. Consider the cost minimization problem of constructing the manufacturing composite with the domestic manufactured good and the foreign composite. In a cost minimizing bundle, the share of expenditure on the domestic good is

$$
\begin{aligned}
\frac{p_{D} \tilde{b}_{D}}{p_{C} \tilde{b}_{C}+p_{D} \tilde{b}_{D}} & =\frac{p_{D} \tilde{b}_{D}}{p_{C} \tilde{b}_{D}\left[\frac{(1-\lambda) p_{D}}{\lambda p_{C}}\right]^{\sigma}+p_{D} \tilde{b}_{D}} \\
& =\frac{p_{D}}{\left[\frac{(1-\lambda) p_{D}}{\lambda p_{C}}\right]^{\sigma}+p_{D}} \\
& =\frac{\lambda^{\sigma} p_{D}^{-(\sigma-1)}}{(1-\lambda)^{\sigma} p_{C}^{-(\sigma-1)}+\lambda^{\sigma} p_{D}^{-(\sigma-1)}} .
\end{aligned}
$$

We now derive the elasticity of domestic demand. The domestic demand can be written 
as

$$
x_{D}=x \cdot \tilde{b}_{D}
$$

where $\tilde{b}_{D}$ is the demand for the domestic good for use in making one unit of the composite manufacturing good and $x$ is the demand for the composite manufacturing good. The elasticity of domestic demand satisfies

$$
\begin{aligned}
\varepsilon_{D} & =-p_{D} \frac{d \ln \left(x_{D}\right)}{d p_{D}} \\
& =-p_{D}\left[\frac{d\left[\ln x+\ln \tilde{b}_{D}\right]}{d p_{D}}\right] \\
& =-p_{D} \frac{d \ln x}{d p_{D}}-p_{D} \frac{d \ln \tilde{b}_{D}}{d p_{D}} .
\end{aligned}
$$

We consider these two terms one at a time:

$$
\begin{aligned}
-p_{D} \frac{d \ln x}{d p_{D}} & =-\frac{p_{D}}{x} \frac{d x}{d p_{x}} \frac{d p_{x}}{d p_{D}} \\
& =-\frac{p_{x}}{x} \frac{d x}{d p_{x}} \frac{p_{D}}{p_{x}} \frac{d p_{x}}{d p_{D}} \\
& =\varepsilon_{x} \frac{p_{D}}{p_{x}} \frac{d p_{x}}{d p_{D}}
\end{aligned}
$$

But recall that

$$
p_{x}=\left[(1-\lambda)^{\sigma} p_{C}^{-(\sigma-1)}+\lambda^{\sigma} p_{D}^{-(\sigma-1)}\right]^{-\frac{1}{\sigma-1}}
$$

so the slope $\frac{d p_{x}}{d p_{D}}$ is

$$
\begin{aligned}
\frac{d p_{x}}{d p_{D}} & =-\frac{1}{(\sigma-1)} \frac{p_{x}}{\left[(1-\lambda)^{\sigma} p_{C}^{-(\sigma-1)}+\lambda^{\sigma} p_{D}^{-(\sigma-1)}\right]}(-1)(\sigma-1) \lambda^{\sigma} p_{D}^{-\sigma} \\
& =\frac{p_{x}}{p_{D}} \frac{\lambda^{\sigma} p_{D}^{-(\sigma-1)}}{\left[(1-\lambda)^{\sigma} p_{C}^{-(\sigma-1)}+\lambda^{\sigma} p_{D}^{-(\sigma-1)}\right]}
\end{aligned}
$$




$$
=\frac{p_{x}}{p_{D}} \frac{p_{D} x_{D}}{p_{C} x_{C}+p_{D} x_{D}}
$$

where the last step uses the formula (45) for the expenditure share. Substituting this into our earlier formula for the first term yields the following expression for the first term:

$$
\begin{aligned}
-p_{D} \frac{d \ln x}{d p_{D}} & =\varepsilon_{x} \frac{p_{D}}{p_{x}} \frac{d p_{x}}{d p_{D}} \\
& =\varepsilon_{x} \frac{p_{D} x_{D}}{p_{C} x_{C}+p_{D} x_{D}} .
\end{aligned}
$$

Now consider the second term:

$$
\begin{aligned}
-p_{D} \frac{d \ln \tilde{b}_{D}}{d p_{D}} & =-p_{D} \frac{1}{\tilde{b}_{D}} \frac{d \tilde{b}_{D}}{d p_{D}} \\
& =-p_{D}\left(-\frac{\sigma}{\sigma-1}\right) \frac{(\sigma-1)(1-\lambda)^{\sigma} \lambda^{-(\sigma-1)} p_{D}^{\sigma-2} p_{C}^{-(\sigma-1)}}{\left[(1-\lambda)\left[\frac{(1-\lambda) p_{D}}{\lambda p_{C}}\right]^{\sigma-1}+\lambda\right]} \\
& =\sigma \frac{(1-\lambda)^{\sigma} \lambda^{-(\sigma-1)} p_{D}^{\sigma-1} p_{C}^{-(\sigma-1)}}{\left[(1-\lambda)\left[\frac{(1-\lambda) p_{D}}{\lambda p_{C}}\right]^{\sigma-1}+\lambda\right]} \\
& =\sigma \frac{(1-\lambda)^{\sigma} p_{C}^{-(\sigma-1)}}{(1-\lambda)^{\sigma} p_{C}^{-(\sigma-1)}+\lambda^{\sigma} p_{D}^{-(\sigma-1)}} \\
& =\sigma \frac{p_{C} x_{C}}{p_{C} x_{C}+p_{D} x_{D}} .
\end{aligned}
$$

We conclude by putting the two terms together. This gives us

$$
\varepsilon_{D}=\varepsilon_{x} \frac{p_{D} x_{D}}{p_{C} x_{C}+p_{D} x_{D}}+\sigma \frac{p_{C} x_{C}}{p_{C} x_{C}+p_{D} x_{D}}
$$

which is the formula reported in the text as equation (25). 


\section{Result for Section 5}

This part of the appendix proves the main proposition stated in Section 5 , namely, $\pi^{* *}(r, \tau)$ is strictly decreasing in $\tau$. As discussed in the text, the ratio can be written as

$$
\text { (48) } \pi=k_{D, 1} \frac{p_{2} x_{D, 2}}{p_{1} x_{D, 1}}+\left(1-k_{D, 1}\right) \frac{p_{2} x_{E, 2}}{p_{1} x_{E, 1}}
$$

where $k_{D, 1}$ is defined to be the following share:

$$
\begin{aligned}
k_{D, 1} & =\frac{p_{1} x_{D, 1}}{p_{1} x_{D, 1}+p_{1} x_{E, 1}} \\
& =\frac{x_{D, 1}}{x_{D, 1}+x_{E, 1}} .
\end{aligned}
$$

To show that (48) is strictly decreasing in $\tau$, we prove four claims. These four claims together are sufficient for the result.

We note that the proof is tedious. The complexity arises because we consider the general $\eta$ case. For the case of $\eta=1$, the derivations are simpler, as will be apparent as we go through the proof.

\section{Step 1}

We first show that under the assumption that $\eta \geq 4-3 \sigma$ made in the proposition, $\frac{p_{2} x_{D, 2}}{p_{1} x_{D, 1}}$ is decreasing in $\tau$. Note that under assumptions (30) and (31), $p_{2}$ and $p_{1}$ are independent of $\tau$, so we must show that $\frac{x_{D, 2}}{x_{D, 1}}$ is decreasing in $\tau$. Also, the effect on this ratio is through its effect on $p_{C}$. So we must show that this ratio is decreasing in $p_{C}$. The ratio can be written as

$$
\frac{x_{D, 2}}{x_{D, 1}}=\frac{x_{2} \cdot \tilde{b}_{D, 2}}{x_{1} \cdot \tilde{b}_{D, 1}} .
$$


It is useful at this point to recall some equations reported in the text:

$$
p_{x}=\left[(1-\lambda)^{\sigma} p_{C}^{-(\sigma-1)}+\lambda^{\sigma} p_{D}^{-(\sigma-1)}\right]^{-\frac{1}{\sigma-1}}
$$

and

$$
\tilde{b}_{D}=\left[(1-\lambda)\left[\frac{(1-\lambda) p_{D}}{\lambda p_{C}}\right]^{\sigma-1}+\lambda\right]^{-\frac{\sigma}{\sigma-1}} .
$$

Manipulating the equation for $\tilde{b}_{D}$ yields

$$
\begin{aligned}
\tilde{b}_{D} & =\left[\lambda^{-(\sigma-1)} p_{D}^{(\sigma-1)}\right]^{-\frac{\sigma}{\sigma-1}}\left[(1-\lambda)^{\sigma} p_{C}^{-(\sigma-1)}+\lambda^{\sigma} p_{D}^{-(\sigma-1)}\right]^{-\frac{\sigma}{\sigma-1}} \\
& =\lambda^{\sigma} p_{D}^{-\sigma} p_{x}^{\sigma}
\end{aligned}
$$

so

$$
\begin{aligned}
\frac{x_{D, 2}}{x_{D, 1}} & =\frac{x_{2} \cdot \tilde{b}_{D, 2}}{x_{1} \cdot \tilde{b}_{D, 1}} \\
& =\frac{\left[\alpha^{\eta}(1-\alpha)^{-\eta} p_{x, 1}^{\eta}+p_{x, 1}\right]}{\left[\alpha^{\eta}(1-\alpha)^{-\eta} p_{x, 2}^{\eta}+p_{x, 2}\right]} \frac{\lambda^{\sigma} p_{D, 2}^{-\sigma} p_{x, 2}^{\sigma}}{\lambda^{\sigma} p_{D, 1}^{-\sigma} p_{x, 1}^{\sigma}} \\
& =\frac{\left[\alpha^{\eta}(1-\alpha)^{-\eta} p_{x, 1}^{\eta}+p_{x, 1}\right]}{\left[\alpha^{\eta}(1-\alpha)^{-\eta} p_{x, 2}^{\eta}+p_{x, 2}\right]} \frac{p_{x, 2}^{\sigma}}{p_{x, 1}^{\sigma}}\left[\frac{p_{D, 2}}{p_{D, 1}}\right]^{-\sigma} \\
& =\frac{\left[\alpha^{\eta}(1-\alpha)^{-\eta} p_{x, 1}^{\eta}+p_{x, 1}\right]}{\left[\alpha^{\eta}(1-\alpha)^{-\eta} p_{x, 2}^{\eta}+p_{x, 2}\right]} \frac{p_{x, 2}^{\sigma}}{p_{x, 1}^{\sigma}} \gamma^{\sigma} .
\end{aligned}
$$

We must determine how this varies with $p_{C}$. It is sufficient to show that

$$
H\left(p_{C}\right)=\frac{\omega p_{x, 1}^{\eta-\sigma}+p_{x, 1}^{1-\sigma}}{\omega p_{x, 2}^{\eta-\sigma}+p_{x, 2}^{1-\sigma}}
$$

is decreasing in $p_{C}$, where

$$
\omega=\alpha^{\eta}(1-\alpha)^{-\eta}
$$


We can write $H\left(p_{C}\right)$ as

$$
H\left(p_{C}\right)=\frac{\omega p_{x, 1}^{\eta-\sigma}+(1-\lambda)^{\sigma} p_{C}^{-(\sigma-1)}+\lambda^{\sigma} p_{D, 1}^{-(\sigma-1)}}{\omega p_{x, 2}^{\eta-\sigma}+(1-\lambda)^{\sigma} p_{C}^{-(\sigma-1)}+\lambda^{\sigma} p_{D, 2}^{-(\sigma-1)}} .
$$

Note that $p_{D, 1}=1$ and $p_{D, 2}=\frac{1}{\gamma}$. Define

$$
z=(1-\lambda)^{\sigma} p_{C}^{-(\sigma-1)} .
$$

In this case,

$$
\begin{aligned}
& p_{x, 1}=\left[z+\lambda^{\sigma}\right]^{-\frac{1}{\sigma-1}} \\
& p_{x, 2}=\left[z+\lambda^{\sigma} \gamma^{\sigma-1}\right]^{-\frac{1}{\sigma-1}}
\end{aligned}
$$

so

$$
\begin{aligned}
H(z) & =\frac{\omega\left[z+\lambda^{\sigma}\right]^{\frac{\sigma-\eta}{\sigma-1}}+z+\lambda^{\sigma}}{\omega\left[z+\lambda^{\sigma} \gamma^{\sigma-1}\right]^{\frac{\sigma-\eta}{\sigma-1}}+z+\lambda^{\sigma} \gamma^{\sigma-1}} \\
& =\frac{\omega[z+\beta]^{\theta}+z+\rho}{\omega[z+\xi]^{\theta}+z+\xi}
\end{aligned}
$$

where

$$
\begin{aligned}
\rho & =\lambda^{\sigma} \\
\xi & =\lambda^{\sigma} \gamma^{\sigma-1}, \text { and } \xi>\rho \\
\theta & =\frac{\sigma-\eta}{\sigma-1}
\end{aligned}
$$

since $\sigma>1$ and $\sigma>\eta, \theta>0$.

We need to show that the above is increasing in $z$. This holds if and only if

$$
\left[\omega \theta[z+\rho]^{\theta-1}+1\right]\left[\omega[z+\xi]^{\theta}+z+\xi\right]
$$




$$
>\left[\omega \theta[z+\xi]^{\theta-1}+1\right]\left[\omega[z+\rho]^{\theta}+z+\rho\right]
$$

or

$$
\frac{\omega[z+\xi]^{\theta}+z+\xi}{\omega[z+\rho]^{\theta}+z+\rho}>\frac{\omega \theta[z+\xi]^{\theta-1}+1}{\omega \theta[z+\rho]^{\theta-1}+1} .
$$

It is immediate that this is true for $\theta=1(\eta=1)$. Suppose that $\theta<1(\eta>1)$. Then $\theta-1<0$. Since $\xi>\rho$, the right-hand side above is less than one. Hence, the inequality holds since the left-hand side is greater than one. So now suppose that $\eta<1$, so $\theta>1$. We need to show that

$$
\frac{\omega[z+\xi]^{\theta}+z+\xi}{\omega \theta[z+\xi]^{\theta-1}+1}>\frac{\omega[z+\rho]^{\theta}+z+\rho}{\omega \theta[z+\rho]^{\theta-1}+1} .
$$

Since $\xi>\rho$, we need to show that

$$
L(h)=\frac{\omega[z+h]^{\theta}+z+h}{\omega \theta[z+h]^{\theta-1}+1}
$$

is strictly increasing in $h$. But $L^{\prime}(h)$ has the sign of

$$
\begin{aligned}
& {\left[\omega \theta[z+h]^{\theta-1}+1\right]\left[\omega \theta[z+h]^{\theta-1}+1\right] } \\
& -\omega \theta(\theta-1)[z+h]^{\theta-2}\left[\omega[z+h]^{\theta}+z+h\right] \\
= & \omega^{2} \theta^{2}[z+h]^{2 \theta-2}+2 \omega \theta[z+h]^{\theta-1}+1 \\
& -\omega^{2} \theta(\theta-1)[z+h]^{2 \theta-2}-\omega \theta(\theta-1)[z+h]^{\theta-1} \\
= & 1+\omega^{2} \theta[z+h]^{2 \theta-2}+\omega \theta(2-\theta+1)[z+h]^{\theta-1} \\
= & 1+\omega^{2} \theta m^{2}+\omega \theta(3-\theta) m
\end{aligned}
$$

for

$$
m=[z+h]^{\theta-1}
$$


To obtain our result, it is sufficient to show that the above is positive for all $m>0$. If $3 \geq \theta$, then the result obviously holds, so assume that $3<\theta$. Note that the function is strictly convex in $m$. Suppose that we minimize the function over $m$. The point $m^{*}$ where the minimum is obtained satisfies the FONC:

$$
2 \omega^{2} \theta m+\omega \theta(3-\theta)=0
$$

or

$$
\begin{aligned}
m^{*} & =\frac{\omega \theta(\theta-3)}{2 \omega^{2} \theta} \\
& =\frac{(\theta-3)}{2 \omega} .
\end{aligned}
$$

Evaluating the function at $m^{*}$ yields

$$
\begin{aligned}
& 1+\omega^{2} \theta m^{* 2}+\omega \theta(3-\theta) m^{*} \\
= & 1+\omega^{2} \theta \frac{(\theta-3)^{2}}{4 \omega^{2}}+\omega \theta(3-\theta) \frac{(\theta-3)}{2 \omega} \\
= & 1+\frac{\theta(\theta-3)^{2}}{4}-\frac{\theta(\theta-3)^{2}}{2} \\
= & 1-\frac{\theta(\theta-3)^{2}}{4} .
\end{aligned}
$$

This will be positive if and only if

$$
4>\theta(\theta-3)^{2}
$$

which holds if $\theta<4$. Hence, our sufficient condition is

$$
4 \geq \theta=\frac{\sigma-\eta}{\sigma-1}
$$


or

$$
\eta \geq 4-3 \sigma
$$

\section{Step 2}

In this step, we show that the domestic revenue share $k_{D, 1}$ is strictly increasing in $\tau$. To show this, it is sufficient to show that $x_{D, 1}$ increases and $x_{E, 1}$ decreases. Note that we are holding $r$ fixed in this comparative statics exercise, so $\mu$ is fixed. The set of products is fixed, and $\tau$ going up obviously implies that $p_{C}$ increases. So it is sufficient to show that $x_{E, 1}$ decreases and that $x_{D, 1}$ increases as $p_{C}$ increases. The fact that $x_{E, 1}$ decreases is immediate. Domestic sales are

$$
x_{D}=x \cdot \tilde{b}_{D}
$$

We need to show that this increases in $p_{C}$. This holds if

$$
\ln x_{D}=\ln x+\ln \tilde{b}_{D}
$$

is increasing in $p_{C}$; that is,

$$
\frac{1}{x} \frac{d x}{d p_{C}}+\frac{1}{\widetilde{b}_{D}} \frac{d \tilde{b}_{D}}{d p_{C}}>0
$$

which holds if and only if

$$
\frac{p_{C}}{x} \frac{d x}{d p_{C}}+\frac{p_{C}}{\tilde{b}_{D}} \frac{d \widetilde{b}_{D}}{d p_{C}}>0
$$

We consider the second term first:

$$
\frac{p_{C}}{\tilde{b}_{D}} \frac{d \tilde{b}_{D}}{d p_{C}}=-\frac{p_{C} \sigma}{\sigma-1} \frac{-(\sigma-1)(1-\lambda)^{\sigma} \lambda^{-(\sigma-1)} p_{D}^{(\sigma-1)} p_{C}^{-\sigma}}{\left[(1-\lambda)\left[\frac{(1-\lambda) p_{D}}{\lambda p_{C}}\right]^{\sigma-1}+\lambda\right]}
$$




$$
\begin{aligned}
& =\sigma \frac{(1-\lambda)^{\sigma} p_{C}^{-(\sigma-1)}}{(1-\lambda)^{\sigma} p_{C}^{-(\sigma-1)}+\lambda^{\sigma} p_{D}^{-(\sigma-1)}} \\
& =\sigma \frac{p_{C} x_{C}}{p_{C} x_{C}+p_{D} x_{D}} .
\end{aligned}
$$

We now turn to the first term:

$$
\frac{p_{C}}{x} \frac{d x}{d p_{C}}=-\frac{p_{C}}{\left[\alpha^{\eta}(1-\alpha)^{\eta}{ }^{-\eta} p_{x}^{\eta} p_{y}^{1-\eta}+p_{x}\right]} \frac{d p_{x}}{d p_{C}}
$$

but using a symmetric argument to that above, we have

$$
\frac{d p_{x}}{d p_{C}}=\frac{p_{x}}{p_{C}} \frac{p_{C} x_{C}}{p_{C} x_{C}+p_{D} x_{D}}
$$

so

$$
\begin{aligned}
\frac{p_{C}}{x} \frac{d x}{d p_{C}} & =-p_{x} \frac{\eta \alpha^{\eta}(1-\alpha)^{-\eta} p_{x}^{\eta-1} p_{y}^{1-\eta}+1}{\left[\alpha^{\eta}(1-\alpha)^{-\eta} p_{x}^{\eta} p_{y}^{1-\eta}+p_{x}\right]} \frac{p_{C} x_{C}}{p_{C} x_{C}+p_{D} x_{D}} \\
& =-\frac{\eta \alpha^{\eta}(1-\alpha)^{-\eta} p_{x}^{\eta} p_{y}^{1-\eta}+p_{x}}{\left[\alpha^{\eta}(1-\alpha)^{-\eta} p_{x}^{\eta} p_{y}^{1-\eta}+p_{x}\right]} \frac{p_{C} x_{C}}{p_{C} x_{C}+p_{D} x_{D}}
\end{aligned}
$$

Our condition is positive if and only if

$$
-\frac{\eta \alpha^{\eta}(1-\alpha)^{-\eta} p_{x}^{\eta} p_{y}^{1-\eta}+p_{x}}{\left[\alpha^{\eta}(1-\alpha)^{-\eta} p_{x}^{\eta} p_{y}^{1-\eta}+p_{x}\right]}+\sigma>0
$$

or

$$
-\varepsilon_{x}+\sigma>0
$$

which holds.

\section{Step 3}

In this step, we show that

$$
\frac{p_{2} x_{D, 2}}{p_{1} x_{D, 1}}<\frac{p_{2} x_{E, 2}}{p_{1} x_{E, 1}} .
$$


This holds if and only if

$$
\frac{x_{D, 2}}{x_{D, 1}}<\frac{x_{E, 2}}{x_{E, 1}}
$$

To show this, note that the ratio of export output on the right-hand side must satisfy the consumer marginal rate of substitution condition

$$
\begin{aligned}
\frac{x_{E, 2}}{x_{E, 1}} & =\left[\frac{p_{E, 2}}{p_{E, 1}}\right]^{-\sigma} \\
& =\left[\frac{p_{D, 2}}{p_{D, 1}}\right]^{-\sigma}
\end{aligned}
$$

Now we know that

$$
\frac{x_{D, 2}}{x_{D, 1}}<\left[\frac{p_{D, 2}}{p_{D, 1}}\right]^{-\sigma} .
$$

because we showed earlier that the elasticity of domestic demand is less than $\sigma$. This completes the proof of Step 3. Q.E.D.

\section{Step 4}

The ratio $\frac{p_{2} x_{E, 2}}{p_{1} x_{E, 1}}$ is constant in $\tau$. This is immediate from the fact that $\frac{p_{2}}{p_{1}}$ is constant in $\tau$. Q.E.D. 


\section{References}

Aghion, Philippe, and Howitt, Peter. "A Model of Growth Through Creative Destruction," Econometrica 60(2), 1992, pp. 323-351.

Baily, Martin Neil, and Gersbach, Hans. "Efficiency in Manufacturing and the Need for Global Competition," Brookings Papers on Economic Activity, Microeconomics 1995, pp. $307-47$.

Balassa, Bela. Trade Liberalization Among Industrialized Countries: Objectives and Alternatives. New York: McGraw-Hill, 1967.

Grossman, Gene M., and Helpman, Elhanan. Innovation and Growth in the Global Economy. Cambridge, Mass: MIT Press, 1991.

Holmes, Thomas J., and Schmitz, James A. Jr. "Resistance to New Technology and Trade Between Areas," Federal Reserve Bank of Minneapolis Quarterly Review 19, Winter, 1995, pp. 2-17.

Leibenstein, Harvey. "Allocative Efficiency vs. "X-Efficiency," American Economic Review 56(3), 1966, pp. 392-415.

Manufacturing Productivity. Washington, D.C.: McKinsey and Company, Inc., 1993.

Olson, Mancur. The Rise and Decline of Nations: Economic Growth, Stagflation and Social Rigidities. New Haven, Conn.: Yale University Press, 1982.

Parente, Stephen L., and Prescott, Edward C. "Monopoly Rights: A Barrier to Riches," Research Department Staff Report 236, Federal Reserve Bank of Minneapolis, 1997. 
Table 1

Multi-Country Equilibrium for High $\sigma$ Case and Various Levels of $\tau$

\begin{tabular}{|c|c|c|c|c|c|}
\hline Variable & Notation & $\begin{array}{c}\tau=\infty \\
\text { (autarky) }\end{array}$ & $\tau=1.85$ & $\tau=.45$ & $\begin{array}{l}\tau=0 \\
\text { (free } \\
\text { trade) }\end{array}$ \\
\hline $\begin{array}{l}\text { Activity Levels } \\
\text { research }\end{array}$ & $r$ & .04 & .82 & .98 & .99 \\
\hline obstruction & $s$ & .96 & .18 & .02 & .01 \\
\hline $\begin{array}{l}\text { Success prob. } \\
\text { research }\end{array}$ & $f(r)$ & .04 & .18 & .20 & .20 \\
\hline obstruction & $g(s)$ & .59 & .25 & .09 & .06 \\
\hline \multicolumn{6}{|l|}{ Prices } \\
\hline Domestic, $a=1$ & $p_{D, 1}$ & 1.00 & 1.00 & .71 & .65 \\
\hline Export, $a=1$ & $p_{E, 1}$ & .63 & .63 & .63 & .63 \\
\hline Domestic, $a=2$ & $p_{D, 2}$ & .50 & .50 & .50 & .41 \\
\hline Export, $a=2$ & $p_{E, 2}$ & .31 & .31 & .31 & .31 \\
\hline \multicolumn{6}{|l|}{ Revenues } \\
\hline Domestic, $a=1$ & $p_{D, 1} x_{D, 1}$ & .30 & .23 & .18 & .06 \\
\hline Export, $a=1$ & $p_{E, 1} x_{E, 1}$ & .00 & .01 & .06 & .07 \\
\hline Domestic, $a=2$ & $p_{D, 2} x_{D, 2}$ & .30 & .29 & .23 & .18 \\
\hline Export, $a=2$ & $p_{E, 2} x_{E, 2}$ & .00 & .10 & .61 & 1.07 \\
\hline Profit ratio & $\pi_{2} / \pi_{1}$ & 1.00 & 1.95 & 5.06 & 10.02 \\
\hline \multicolumn{6}{|l|}{ Rung proportions } \\
\hline$a=0$ & $\mu_{0}$ & .54 & .54 & .54 & .54 \\
\hline$a=1$ & $\mu_{1}$ & .36 & .27 & .26 & .25 \\
\hline$a=2$ & $\mu_{2}$ & .10 & .19 & .20 & .21 \\
\hline
\end{tabular}


Figure 1

Equilibrium of Research/Obstruction Game

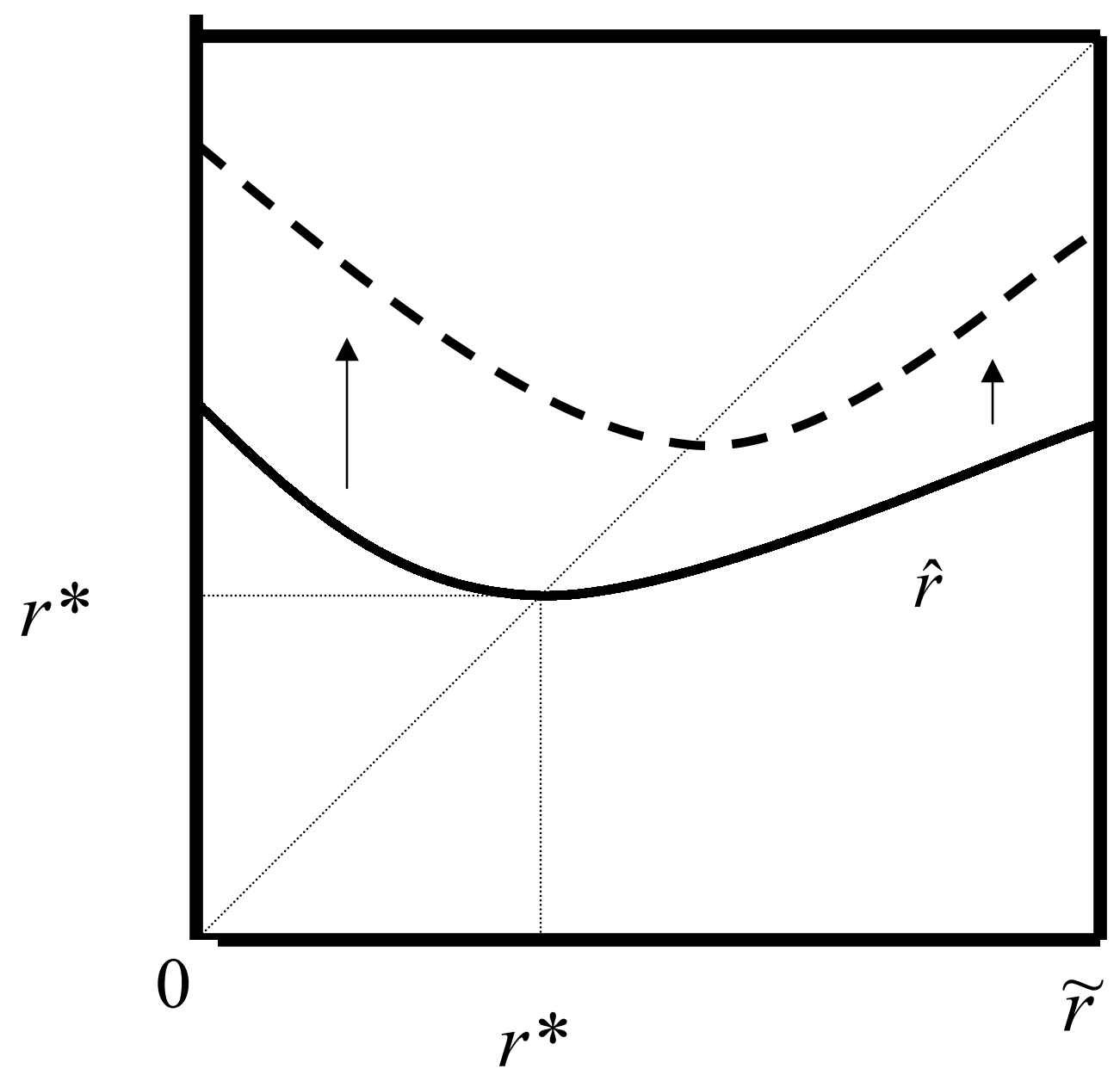


Figure 2

Illustration of $r^{*}$ and $\pi^{* *}$ curves

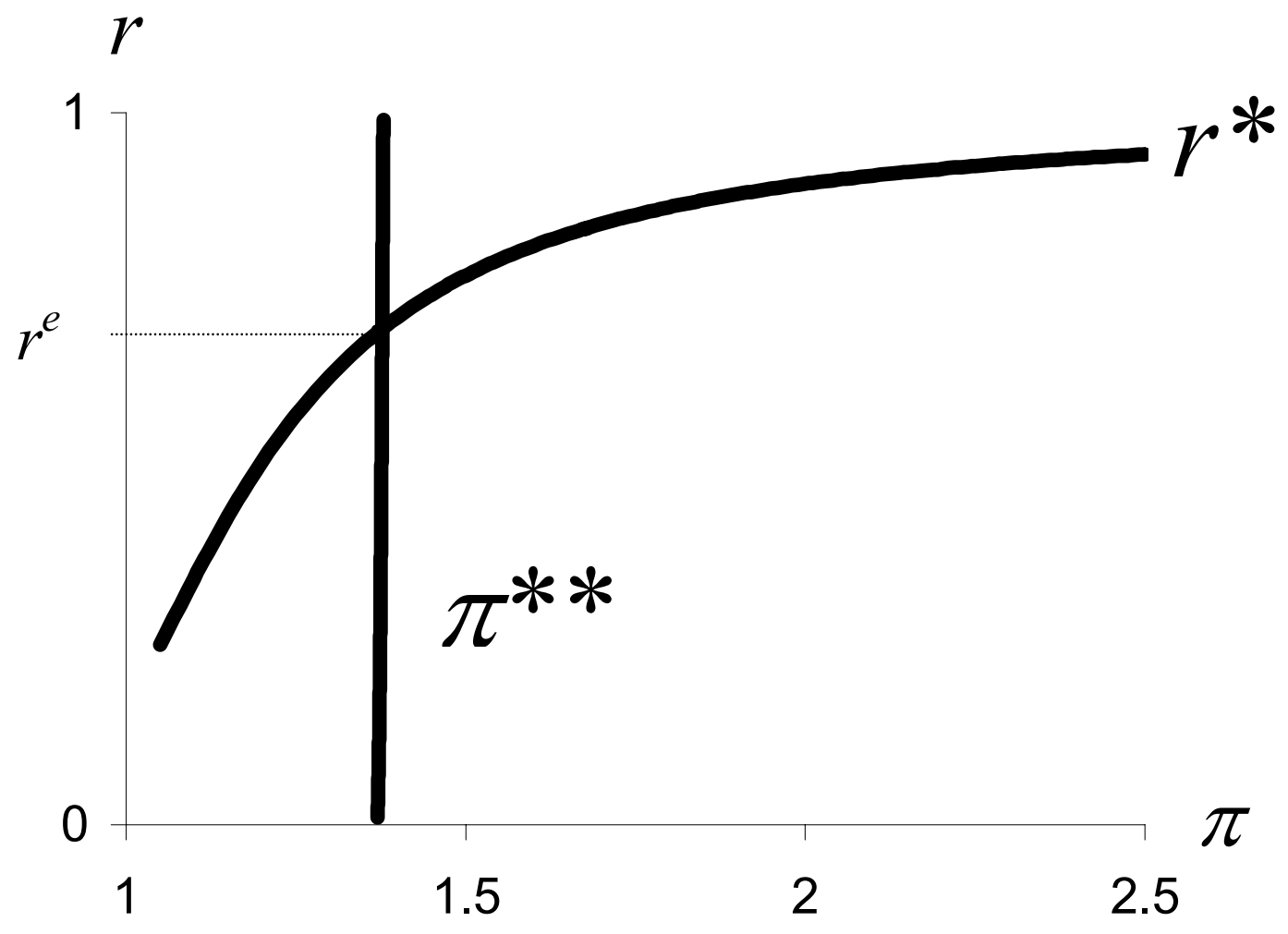


Figure 3

Effect of a Decrease in the Tariff on the $r^{*}$ Curve and $r^{e}$

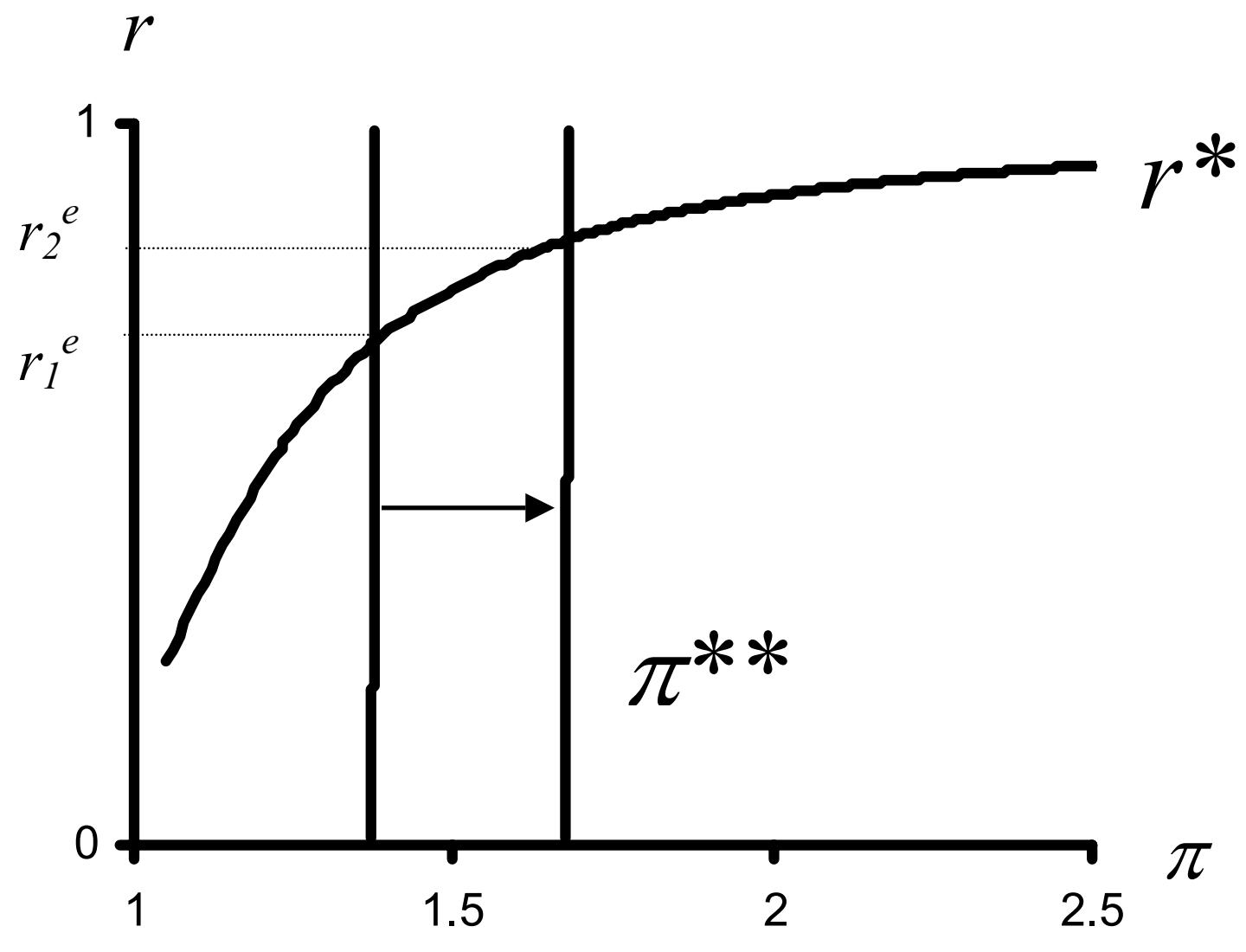

